\title{
Cyclic N-2-Chloroethyl-sulfamide Compounds with a Phosphonate Moiety: Synthesis, Characterization, X-Ray Crystallographic Study and Antimicrobial Evaluation
}

\author{
Zineb Aouf, *[a] Sofiane Bouacida, ${ }^{[b, c]}$ Chahrazed Benzaid, ${ }^{[d]}$ Aïcha Amira, ${ }^{[a]}$ Hacène K'tir, ${ }^{[a]}$ Monique \\ Mathé-Allainmat, ${ }^{[e]}$ Jacques Lebreton, ${ }^{[e]}$ Nour-Eddine Aouf ${ }^{[a]}$
}

\begin{abstract}
[a] Dr. Zineb AOUF, Dr. Aïcha AMIRA, Dr. Hacène K'tir, Pr. N-E, AOUF Chemistry Department

Laboratory of Applied Organic Chemistry, Bioorganic Chemistry Group

Badji Mokhtar-Annaba University, Box 12, 23000 Annaba, Algeria.

E-mail: aouf.zineb27@gmail.com
\end{abstract}

[b] and [c] Pr. Sofiane Bouacida

Department of Materials Science,

Larbi Ben Mhidi University, Oum El Bouaghi 04000, Algeria.

Chemistry Department

Research Unit for Chemistry of the Environment and Molecular Structural

University of Constantine 1, Constantine 25000, Algeria

[d] Dr. Chahrazed Benzaid

Biochemistry Department

Biofilms and Biocontamination of Materials Research group

Badji-Mokhtar-Annaba University, Box 12, 23000 Annaba Algeria.

[e] Dr. Monique Mathé-Allainmat, Pr. Jacques Lebreton

Chimie et Interdisciplinarité: Synthèse, Analyse, Modélisation (CEISAM)

Université de Nantes, CNRS n 6230, 2, rue de la Houssinière-BP 92208-44322 NANTES Cedex 3, France.

\begin{abstract}
An efficient access to a novel series of organophosphorus compounds with a cyclosulfamide moiety is described. Starting from $\alpha$-hydroxyphosphonates, the 2-chloroethyl-(1, 2, 5- thiadiazoline-2carbonyloxy-1- dioxide) phosphonates compounds were prepared in three steps including an abramov reaction, carbamoylationsulfamoylation with chlorosulfonyl isocyanate and an intramolecular cyclisation. The spectroscopic methods $\left({ }^{1} \mathrm{H}\right.$ NMR, ${ }^{13} \mathrm{C}$ NMR, ${ }^{31} \mathrm{P}$ NMR, $\mathrm{MS}$ and IR) were used to determine the structural characteristics of the synthesized compounds and X-ray crystallographic data were provided for compound $3 c$. Some selected compounds were screened in vitro for their antimicrobial activity against a panel of microorganisms, resulting in a good antibacterial activity compared to standard drugs.
\end{abstract}

\section{Introduction}

Sulfamides compounds bearing a phosphonate moiety have emerged as attractive chemical targets, during the last decades, due to their structural and biological advantages. The sulfamide group has appeared as a good bioisostere of sulfonamide ${ }^{[1]}$ or urea ${ }^{[2-3]}$ and in drug analogs, it can enhance physico-chemical properties such as water solubility and bioavailability.

Moreover the geometry of both nitrogen atoms as well as the oxygen atoms at the tetrahedral sulfur can be in favor of electrostatic and hydrogen bond interactions to the targeted proteins. ${ }^{4}$ Consequently the biological efficiency of sulfamide compounds as enzyme inhibitors, ${ }^{[5-6]}$ such as HIV protease, ${ }^{[7]}$ as well as agonist or antagonist ligands of receptors has led some compounds to the market such as Norprolac (Fig 1, compound 1) used in prolactin-dependent disorder or Famotidine for the treatment of ulcers. ${ }^{[8]}$
In the same manner, cyclosulfamide derivatives or $(2,5-$ thiadiazolidine-1,1-dioxide) are considered as sulfonated analogs of cyclic urea compounds, ${ }^{[9]}$ and have given access to attractive molecules with potential application in medicinal chemistry. ${ }^{[10]}$

Indeed, cyclosulfamides offered a wide range of biological activities such as antibacterial ${ }^{[11]}$ or protease inhibition of HIV-1 ${ }^{[12]}$ and $\mathrm{Y}$-secretase, ${ }^{[13]}$ norovirus inhibitory activity ${ }^{[14]}$ (Fig 1, compound 2).

Bifunctional compounds containing both sulfamide and phosphonate moieties have not been so extensively studied compared to their sulfonamido-phosphonate parents. ${ }^{15-20]}$ However they have also been characterized as promising anticancer agent such as Fotemustine analog $3^{[21]}$ or as potent insecticidal ${ }^{[22]}$ such as compound 4 (Figure 1).

Because sulfonamide and sulfamide compounds have been widely studied for their antimicrobial activities some sulfonamidocarbonyloxymethylphosphonates were also recently developed in our laboratory, as potential antimicrobial agents against Gram-positive and negative bacteria and have shown significant antibacterial activity [23] compared to sulfamethoxazole/trimethoprim as a reference (Figure 1, compound 5).

During the course of our research works, we have also developed several strategies for the synthesis of sulfamidophosphonates compounds. Belhani et al ${ }^{[24]}$ reported a simple and new method for the synthesis of a novel $\alpha$-sulfamidophosphonates (Fig 2, compound 6) via Kabachnik-Fields reaction under ultrasonic (US) 
activation and solvent-free conditions with good yields and short reaction times.

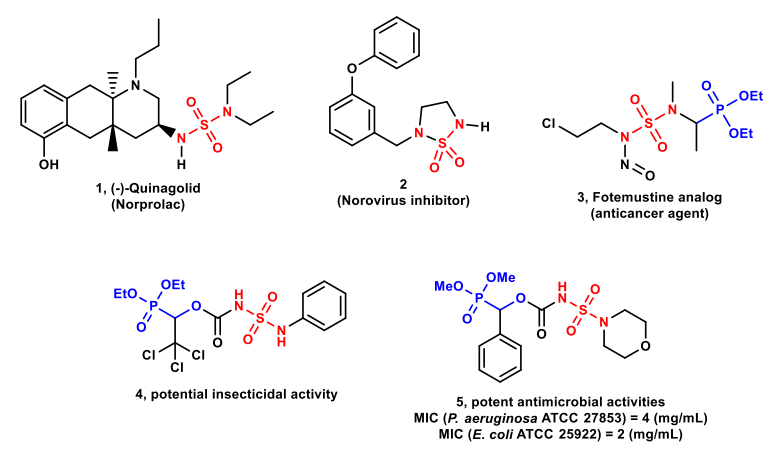

Figure 1. Some examples sulfamide and sulfamidophosphonates compounds with biological interest

In 2017, Bouzina and co-workers [25] realised the phosphorylation of a series of $\mathrm{N}$-acylsulfonamides with trimethylphosphite under US conditions and this process provided in 15 minutes a good yield of product 7 (Fig 2, 88\%). If we considered the concomitant role of cyclosulfamide and phosphonate groups in a single molecule, only few syntheses are reported in the literature. Hessania et al ${ }^{[26]}$ have developed an efficient process for the synthesis of novel phosphonoalkylcyclosulfamide (Fig 2, compound 8), by sulfonylation of diamine with sulfonyl chloride. The synthesis and in vitro cytotoxic activities of new sulfamidophosphonates (Fig 2, compound 9) using cyclic-sulfamidate, analog of cyclosulfamide, was more recently studied by our group. [27]

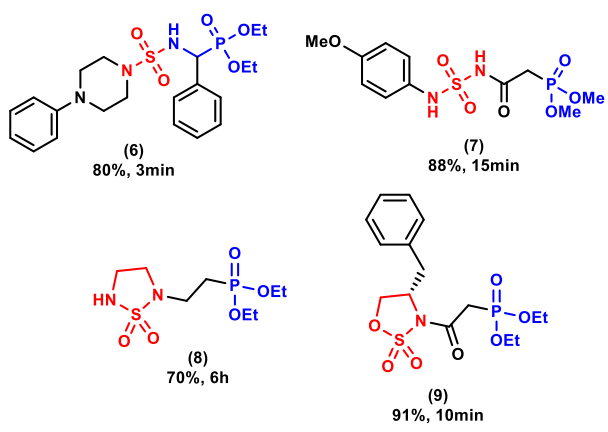

Figure 2. Previous work carried out in our laboratory

In our continuing investigation to the synthesis of new phosphonate derivatives inspired by our previous works, ${ }^{[28]}$ we report in this paper the synthesis and the antimicrobial evaluation of a new series of 5-(2-chloroethyl)-1,2,5-thiadiazolidine-2carbaldehyde-1,1-dioxide tethered to a terminal phosphonate moiety with a substituted carbonyloxymethyl linker (Figure 3).

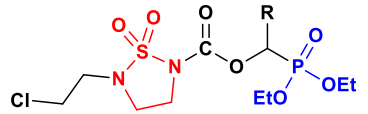

R: Aryl (H, naphtyl, 4-Cl, 2-F, 4-OMe) or thiophen-2-yl \& furan-2-yl

Figure 3. General structure of 5-(2-chloroethyl)-1,2,5-thiadiazolidine-2carbaldehyde-1,1-dioxide with phosphonates moiety

\section{Results and Discussion}

The synthesis of 5-(2-chloroethyl)-1,2,5-thiadiazolidine-2carbaldehyde-1,1-dioxide with phosphonates derivatives 1c-7c was achieved according to the pathway illustrated in (scheme 1). This strategy was carried out in three steps, starting from commercially available reagents (aldehydes, dialkylphosphite, chlorosulfonyl isocyanate (CSI) and bis (2-chloroethyl) amine hydrochloride).

In a first step, the synthesis of the key $\alpha$ hydroxyphosphonates intermediates was achieved via Abramov reaction, [29] in solvent free conditions and with a recyclable catalyst, the heteropolyacid HPA following our method recently described. ${ }^{[30]}$ A series of $\alpha$-hydroxyphosphonates (1a-7a) were prepared involving several aromatic aldehydes (Table 1). The conversions were total within 10 minutes and the phosphonates purified in good to excellent yields. Secondly, carbamoylation of these $\alpha$-hydroxyphosphonates at $0^{\circ} \mathrm{C}$, under anhydrous conditions with commercial chlorosulfonyl isocyanate (CSI), afforded the intermediate compounds ( $\mathrm{N}$-chlorosulfonyl carbamates) in 30 minutes. The crudes were slowly added to a solution of the hydrochloride form of bis-(2-chloroethyl) amine in the presence of triethylamine at $0^{\circ} \mathrm{C}$, to give the sulfamidocarbonyloxymethylphosphonates derivatives $\mathbf{1 b}-\mathbf{7 b}$ in good yields (78-92\%) within 2 hours (Table 1).

Finally, the intramolecular cyclization of these sulfamides (1 $\mathbf{b}$ 7b) was obtained using $\mathrm{K}_{2} \mathrm{CO}_{3}$ as the base in refluxing acetonitrile for 2 hours. The high acidity of the proton of the $\mathrm{N}$ sulfonylcarbamate group is in favor of the expected intramolecular cyclization, and this alkylation step afforded the corresponding cyclic compounds (1c-7c) with quantitative yield (Table 1).

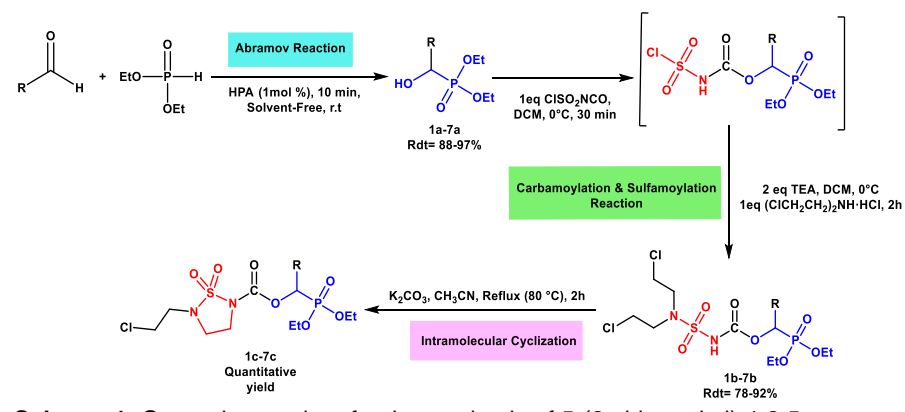

Scheme 1. General procedure for the synthesis of 5-(2-chloroethyl)-1,2,5thiadiazolidine-2-carbaldehyde-1,1-dioxide with phosphonates derivatives 
Table 1. Structures of compounds (1a-7a), (1b-7b) and (1c-7c)

\begin{tabular}{ccc}
\hline a-Hydroxy & Sulfamidocarbonyloxy & 5-(2-Chloroethyl)-1, 2, 5- thiadiazoline-2- \\
phosphonates & methylphosphonates & carboxylate-1, 1-dioxide phosphonates \\
$\mathbf{( 1 a - 7 a )}$ & $(\mathbf{1 b - 7 b )}$ & $(1 \mathrm{c}-7 \mathrm{c})^{\mathrm{c}}$
\end{tabular}<smiles>CCOP(=O)(OCC)C(O)c1ccccc1</smiles>

1 a, $97 \%$<smiles>CCOP(=O)(OCC)C(O)c1ccc(Cl)cc1</smiles>

2a, $93 \%$<smiles>CCOP(=O)(OCC)C(O)c1ccccc1F</smiles>

3a, $90 \%$<smiles>CCOP(=O)(OCC)C(O)c1ccc(OC)cc1</smiles>

4 a, $93 \%$<smiles>CCO[P+](=O)C(O)c1cccc2ccccc12</smiles>

$5 a, 95 \%$<smiles>CCOP(=O)(OCC)C(O)c1cccs1</smiles>

6a, $89 \%$<smiles>CCOP(=O)(OCC)C(O)c1ccco1</smiles>

$7 a, 88 \%$<smiles>CCCCN(C(=O)O)S(=O)(=O)NC(=O)OC(C(=O)O)c1ccccc1</smiles>

1b, $92 \%$<smiles>CCOP(=O)(OCC)C(OC(=O)NS(=O)(=O)N(CCCl)CCCl)c1ccc(Cl)cc1</smiles>

$2 b, 87 \%$ b<smiles>CCOP(=O)(OCC)C(OC(=O)NS(=O)(=O)N(CCCl)CCCl)c1ccccc1F</smiles>

$3 b, 83 \%$ a<smiles>CCOP(=O)(OCC)C(OC(=O)NS(=O)(=O)N(CCCl)CCCl)c1ccc(OC)cc1</smiles>

$4 b, 88 \%$ b<smiles>CCOP(=O)(OC)C(OC(=O)NS(=O)(=O)N(CCCl)CCCl)c1cccc2ccccc12</smiles>

5 b, $90 \%$ a<smiles>CCOP(=O)(OCC)C(OC(=O)NS(=O)(=O)N(CCCl)CCCl)c1cccs1</smiles>

$6 b, 80 \%$<smiles>CCOP(=O)(OCC)C(OC(=O)NS(=O)(=O)N(CCCl)CCCl)c1ccco1</smiles>

$7 b, 78 \%$ b<smiles>O=C(O)C(=O)OC(OC(=O)C1CCN(CCO)C1=O)c1ccccc1</smiles>

$1 c$<smiles>CCOP(=O)(OCC)C(OC(=O)N1CCN(CCCl)S1(=O)=O)c1ccc(Cl)cc1</smiles>

2c<smiles>CCOP(=O)(OCC)C(OC(=O)N1CCN(CCCl)S1(=O)=O)c1ccccc1F</smiles>

$3 c$<smiles>CCOP(=O)(OCC)C(OC(=O)N1CCN(CCCl)S1(=O)=O)c1ccc(OC)cc1</smiles>

4c<smiles>CCOP(=O)(OCC)C(OC(=O)N1CCN(CCCl)S1(=O)=O)c1cccc2ccccc12</smiles>

$5 c$<smiles>CCOP(=O)(OCC)C(OC(=O)N1CCN(CCCl)S1(=O)=O)c1cccs1</smiles>

$6 c$<smiles>CCOP(=O)(OCC)C(OC(=O)N1CCN(CCCl)S1(=O)=O)c1ccco1</smiles>

$7 c$

[a] Purification by column chromatography $\left(\mathrm{CH}_{2} \mathrm{Cl}_{2}\right.$ : $\left.\mathrm{MeOH}(9.8: 0.2)\right)$, [b] Recrystallization in diethyl ether/n-hexane (1/1), [c] Quantitatively obtained with good purity by simple extraction

The

structures

of

the

sulfamidocarbonyloxymethylphosphonates $\mathbf{1 b} \mathbf{b} \mathbf{7 b}$, as well as their cyclic analogues 1c-7c were confirmed by usual spectroscopic methods. The infrared spectra of compounds $\mathbf{1} \mathbf{b}-\mathbf{7} \mathbf{b}$, exhibited characteristic signals for the $\mathrm{NH}$ group (range $3285-3260 \mathrm{~cm}^{-1}$ ), the phosphonate $\left(\mathrm{P}=\mathrm{O}, 1255-1234 \mathrm{~cm}^{-1}\right)$, and the carbonyl $(\mathrm{C}=\mathrm{O}$, 1750-1730 $\mathrm{cm}^{-1}$ ) groups, as well as specific bands for symmetric/antisymmetric sulfonyl function $\mathrm{SO}_{2} \quad(1185 \pm 10$

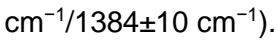

The most significant change in the IR spectra data of cyclic compounds 1c-7c was the disappearance of $\mathrm{NH}$ stretching band vibration.
In the ${ }^{1} \mathrm{H}-\mathrm{NMR}$ spectra of the compounds $\mathbf{1 b}-\mathbf{7 b}$, the $\mathrm{P}-\mathrm{CH}$ gave a doublet in a region between 5.89 and 6.33 ppm (with $J_{H-P}$ $\sim 13.5-16.0 \mathrm{~Hz}$ ) and the carbamic NH proton appeared as a broad singlet at 9.58-10.62 ppm. The two triplets at 1.03-1.38 ppm attributed to the ethoxy groups of the phosphonate moiety as well as the four multiplets integrating for the twelve methylene protons of the phosphonate and the chloroethyl chains (3.52-4.37 ppm) also confirmed the formation of the targeted compounds. For the cyclic products 1c-7c, the absence of the carbamic proton signal and the appearance of two triplets attributed to the methylenes of the cyclosulfamide moiety (3.65-3.45 ppm) were in accordance with an intramolecular cyclization.

The ${ }^{13} \mathrm{C}$ NMR spectra of compounds $\mathbf{1 b} \mathbf{b} \mathbf{7 b}$ mainly showed the presence of the carbonyl group (150.6 ppm) and in the area 
of 64.7-71.7 ppm a doublet with an large coupling constant $\left(J_{C-P}\right.$ $\sim 174.0-189.0 \mathrm{~Hz}$ ) as a characteristic of the trisubstituted carbon in the alpha position of the phosphore. For 1c-7c, the appearance of four signals between 43 and 45 ppm corresponding to the methylene groups of nitrogen mustard pattern confirmed the formation of the five ring membered $N$-substituted 1,2,5thiadiazolidine-1,1-dioxide. All these compounds were finally fully characterized with complementary DEPT and HSQC analyses as well as ${ }^{31} \mathrm{P}$ NMR spectra which confirmed the presence of the phosphonate function (13.0-16.0 ppm), and mass spectrometry were also in good accordance with the products formula.

Satisfyingly, we could obtain a single crystal of the fluoro compound $\mathbf{3 c}$ which was submitted to $\mathrm{X}$-ray diffraction analysis. An ORTEP diagram of structure $3 c$ is shown in figure 4 .

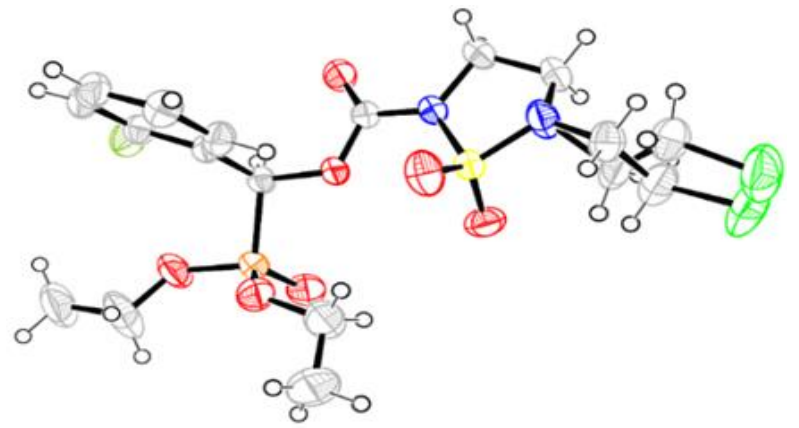

Figure 4. ORTEP diagram of compound 3c displacement ellipsoids are drawn at the $50 \%$ probability level. $\mathrm{H}$ atoms are represented as small spheres of arbitrary radius. (With disordered chloroethyl)

Structural resolution revealed that the asymmetric unit consists of one molecule of (IUPAC NAME), which crystallizes in the triclinic crystal system with $\mathrm{p}-1$ space group (Table 2).
Table 2. Crystallographic data and refinement parameters for $3 \mathrm{c}$

\begin{tabular}{|c|c|}
\hline Formula & $\mathrm{C} 16 \mathrm{H} 23 \mathrm{Cl} F \mathrm{~N} 2 \mathrm{O} 7 \mathrm{P} \mathrm{S}$ \\
\hline Formula weight & 472.84 \\
\hline Crystal habit, color & Prism, Colorless \\
\hline Crystal system & Triclinic \\
\hline Space group & $P-1$ \\
\hline $\mathrm{a}(\AA)$ & $8.686(2)$ \\
\hline$b(\AA)$ & $9.735(3)$ \\
\hline$c(\AA)$ & $13.705(4)$ \\
\hline$\alpha\left(^{\circ}\right)$ & $86.165(14)$ \\
\hline$\beta\left({ }^{\circ}\right)$ & $82.181(13)$ \\
\hline$Y\left({ }^{\circ}\right)$ & $70.724(13)$ \\
\hline Volume (Å3) & $1083.4(5)$ \\
\hline Z, Z' & 2,2 \\
\hline Density (calculated, g cm-3) & 1.446 \\
\hline Absorption coefficient (mm-1) & 0.394 \\
\hline$F(000)$ & 1680 \\
\hline Crystal size (mm) & $0.11 \times 0.15 \times 0.18$ \\
\hline$\theta$ range for data collection $\left(^{\circ}\right)$ & $2.217-38.538$ \\
\hline Reflections collected & 11946 \\
\hline Independent reflections & 6115 \\
\hline Rint & 0.0630 \\
\hline Reflections with $I \geq 2 \sigma(I)$ & 6115 \\
\hline Number of parameters & 290 \\
\hline Goodness-of-fit on F2 & 1.043 \\
\hline Final $R$ indices $[I \geq 2 \sigma(I)]$ & $\mathrm{R} 1=0.0628, w R 2=0.1852$ \\
\hline$R$ indices [all data] & $\mathrm{R} 1=0.1155, \mathrm{wR} 2=0.2128$ \\
\hline Largest difference peak and hole $(\AA-3)$ & $0.583,-0.394$ \\
\hline CCDC deposition no. & CCDC 1986194 \\
\hline
\end{tabular}

The structure was refined with disordered chloroethyl with 64:36 ratio. The crystal packing can be described as alternating layers parallel to $(b, c)$ plane which are connected together with $\mathrm{C}-\mathrm{H}$... $\mathrm{O}$ and $\mathrm{C}-\mathrm{H}$... Cl hydrogen bonds along the a and $\mathrm{c}$ axis (Table 3, Figure 5).

Table 3. Distances $(\AA)$ and angles $\left(^{\circ}\right)$ of hydrogen bond for 3c

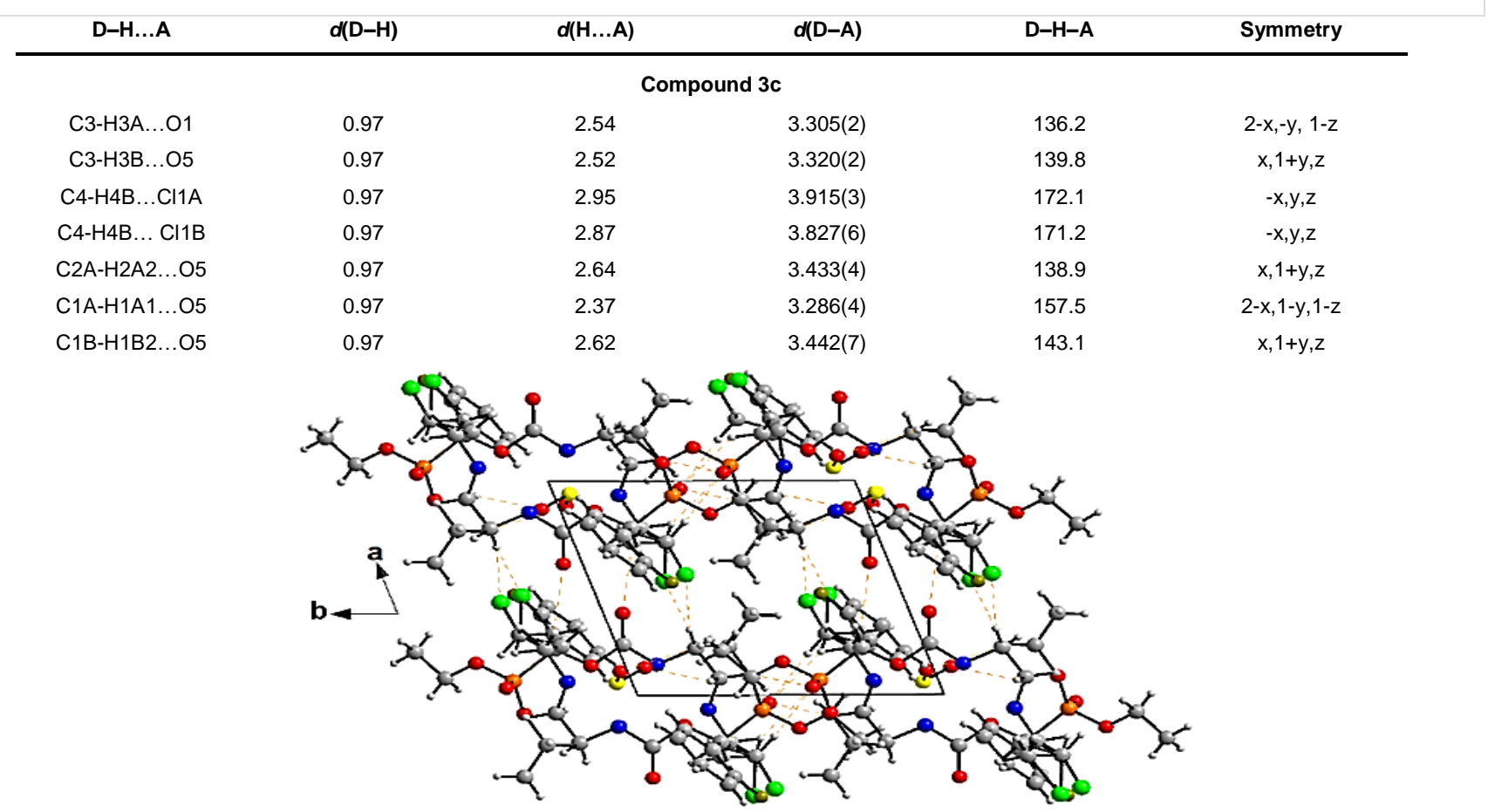

Figure 5. Diagram packing of $3 c$ viewed along the $\mathrm{c}$ axis showing layers parallel to $(\mathrm{b}, \mathrm{c})$ and connected with $\mathrm{C}-\mathrm{H}$..O and $\mathrm{C}-\mathrm{H}$...Cl interactions 
In these layers, the arrangement of each molecule induces a strong $\pi-\pi$ staking intermolecular interactions. The distance centroid-centroid is 3.7431(12) $\AA$ between phenyl rings in the packing crystal, they are oriented face to face with slippage value of $1.730 \AA$.

Our compounds showed interesting antimicrobial activities on the tested microorganisms. The diameters of inhibition zones range from 10 to $30 \mathrm{~mm}$, compared to an inhibition diameter ranging from 10 to 34 with the standard drugs (gentamycin and amphoterin B). These molecules did not discriminated Gram positive or Gram negative strains, and some also appeared effective on Candida albicans (yeasts) showing antifungal activity such as $\mathbf{2 b}, \mathbf{6 b}$ and $\mathbf{5 c}$ (Table 4 ).

In vitro antibacterial activity being confirmed in the disk diffusion test, the Minimum Inhibition Concentrations (MIC) were than determined. As shown in table 4, all the molecules exhibited good activity between 4 and $0.25 \mu \mathrm{g} / \mathrm{ml}$ for all strains compared to the tested treatment (MIC varied between 8 and $1 \mu \mathrm{g} / \mathrm{ml}$ ).

Particularly a MIC of $0.25 \mu \mathrm{g} / \mathrm{ml}$ was observed for compounds 2b, $\mathbf{4 b}$ on E. coli BMR and for compounds $\mathbf{3 c}$ and $\mathbf{5 c}$ on E. coli ATCC 25922.

For sulfonamides $\mathbf{2 b}$ to $\mathbf{7 b}$ bearing the $\mathrm{N}$-bis choroethyl terminal moiety, a lipophilic aromatic substituent in the alpha position of the phosphonate function seemed to be preferred on S.aureus ATCC 29213. As observed, compound $\mathbf{2 b}$ with a $p$ chorophenyl substituent was 8 to 16 time more active than the corresponding thiophen analog $\mathbf{6 b}$ or $p$-methoxyphenyl analog $\mathbf{4 b}$, respectively (Table 5).
It is also to be noted that cyclic compound $\mathbf{5 c}$, bearing a naphthalene substituent conserved the highest activity on a panel of 5 microorganisms (MIC: 0.25 and $0.5 \mu \mathrm{g} / \mathrm{ml}$ ), compared to the cyclic analog 1c bearing a phenyl substituent (MIC: 1 to $4 \mu \mathrm{g} / \mathrm{ml}$ ). Moreover, this thiadiazolidine dioxide derivative $5 \mathrm{c}$ appeared also eight fold more potent than the cyclic morpholino analog $\mathbf{5}$, recently published (Fig 1, compound 5).

\section{Conclusion}

In this work, a series of seven $\mathrm{N}, \mathrm{N}$-bis (2-chloroethyl) sulfamido carbonyloxymethylphosphonate derivatives (1) $\mathbf{b}-\mathbf{7 b})$ and their cyclic analogs (1c-7c) were synthesized by a carbamoylationsulfamoylation reaction of seven $\alpha$-hydroxyphosphonates, followed by intramolecular cyclization. The impact of the aromatic substituent in the alpha position of the phosphonate function for compounds $\mathbf{1 b}-\mathbf{7 b}$ and $\mathbf{1 c - 7 c}$ could so be estimated in the biological tests. All the products isolated were fully characterized by spectroscopic methods, supported with RXD analysis of the compound 3c. In vitro antimicrobial activity was studied against eight microbial strains using the disk diffusion and MIC test. The results revealed that these compounds showed a remarkable antimicrobial activities against Gram-positive, Gram-negative bacteria and fungi at low concentrations. Theoretical studies (DFT and docking study) are underway to explore the interactions of these compounds with biological receptors and extended biological studies (anti-diabetics and anti-cancer) could be carried out to identify the pharmacological potential of our products.

Table 4. Antimicrobial activities of the synthetic compounds a

\begin{tabular}{|c|c|c|c|c|c|c|c|c|c|}
\hline \multicolumn{10}{|c|}{ Diameter of growth inhibition expressed in $\mathbf{m m}$} \\
\hline Microorganism & Acetone & $2 b$ & $4 b$ & $6 b$ & $7 b$ & 1c & $3 c$ & $5 c$ & Treatment \\
\hline K. pneumonaie BLSE & 11 & 24 & 20 & 19 & 23 & 24 & 23,5 & 19 & 29 \\
\hline E. coli BMR & 00 & 22 & 23,5 & 22,5 & 20 & 25 & 25 & 18 & 29 \\
\hline E. coli ATCC 25922 & 05 & 22,5 & 23 & 10 & 00 & 18,5 & 21 & 11 & 30,5 \\
\hline P.areuginosa & 12 & 12,5 & 11,5 & 18 & 20 & 25 & 21 & 10 & 00 \\
\hline P.aeruginosa ATCC27853 & 00 & 15 & 12 & 19 & 17 & 15 & 11,5 & 19 & 10 \\
\hline S. aureus & 05 & 10 & 10 & 00 & 00 & 00 & 16 & 00 & 34 \\
\hline S. aureus ATCC 29213 & 00 & 20 & 11 & 21 & 20 & 20 & 22 & 18 & 15 \\
\hline C. albicans & 10 & 25 & 00 & 30 & 00 & 00 & 00 & 22 & 00 \\
\hline
\end{tabular}

[a] All tests were performed in triplicate

Table 5. The minimal inhibitory concentrations (MIC) a

\begin{tabular}{|c|c|c|c|c|c|c|c|c|}
\hline \multirow[b]{2}{*}{ Micro organism } & \multicolumn{8}{|c|}{ Values MIC $(\mu \mathrm{g} / \mathrm{ml})$} \\
\hline & $2 b$ & $4 b$ & $6 b$ & $7 b$ & 1c & $3 c$ & $5 c$ & Treatment \\
\hline K. pneumonaie BLSE & 2 & 0.5 & 4 & 2 & 2 & 2 & 4 & $\mathrm{R}$ \\
\hline E. coli BMR & 4 & 4 & 4 & 4 & 4 & 4 & 0.5 & 1 \\
\hline E. coli ATCC 25922 & 2 & 0.5 & 2 & 4 & 2 & 4 & 0.5 & 4 \\
\hline P.areuginosa & 2 & 2 & 4 & l & 4 & 0.25 & 0.25 & 4 \\
\hline P.aeruginosa ATCC27853 & 0.25 & 0.25 & 0.5 & 1 & 2 & 2 & 0.5 & 8 \\
\hline S. aureus & 0.25 & 4 & 2 & 1 & 1 & 1 & 0.5 & 8 \\
\hline S. aureus ATCC 29213 & 4 & 4 & l & l & I & 4 & I & 1 \\
\hline C. albicans & 2 & / & 1 & I & I & 0.5 & I & $\mathrm{R}$ \\
\hline
\end{tabular}

[a] All tests were performed in triplicate and sulfamethoxazole-trimethoprim (SXT) was used as a positive control $(\mathrm{MIC}=25 \mu \mathrm{g} / \mathrm{ml})$ 


\section{Experimental Section}

The nomenclature of all compounds is performed according to the international denomination of IUPAC; the reagents were obtained from commercial furnisher (Sigma-Aldrich, Alfa Aesar) and used without further purification

TLC monitored all reactions on silica Merck60 F254 percolated aluminum plates, revealed with UV $(\lambda=254 \mathrm{~nm})$ and dipping in Ninhydrine solution followed by heating. Melting points of all compounds were measured in open capillary tubes using Stuart ${ }^{\mathrm{TM}}$ melting point apparatus SMP3.

IR spectra were recorded on a Perkin-Elmer FT-600 spectrometer. ${ }^{1} \mathrm{HNMR}$ ${ }^{13} \mathrm{C} N M R,{ }^{31} \mathrm{P} N M R$, DEPT, COSY, and HMQC spectra were recorded at $25{ }^{\circ} \mathrm{C}$ with a Bruker spectrometers at 300 or $400,100,161.9 \mathrm{MHz}$, respectively using $\mathrm{CDCl}_{3}$ as solvent with TMS as an internal standard. Chemical displacement values $(\delta)$ are expressed in parts per million (ppm) and coupling constants $(\mathcal{J})$ in Hertz $(\mathrm{Hz})$. Multiplicity of signals is indicated as following: s (singulet), $\mathrm{d}$ (doublet), $\mathrm{t}$ (triplet), q (quadruplet), $\mathrm{m}$ (multiplet) brs (broad singulet) ...etc.

Low-resolution mass spectra (MS) and High-Resolution Mass Spectrometry (HRMS) were performed in CEISAM laboratory on a Waters Xevo GL-XS Qtof spectrometer coupled with an Acquity H-Class LC apparatus and the ionization sources were performed with electrospray ionization $\left(\mathrm{ESI}^{+}\right)$method.

The X-ray single-crystal diffraction was performed on a Brüker APEXII diffractometer and the supplemental materials comprises the characterization data of all news products.

\subsection{General procedure for the synthesis of $\alpha$-hydroxyphosphonates derivatives (1a-7a)}

According to previous work, ${ }^{[30]}$ we prepared the $\alpha$-hydroxyphosphonates from diethylphosphite $(1 \mathrm{mmol})$ and several aromatic aldehydes $(1 \mathrm{mmol})$ at room temperature in the presence of $\mathrm{H}_{6} \mathrm{P}_{2} \mathrm{~W}_{18} \mathrm{O}_{62 .} 14 \mathrm{H}_{2} \mathrm{O}(1 \mathrm{~mol} \%)$ as catalyst under solvent-free conditions for $10 \mathrm{~min}$. Column chromatography on silica gel eluted with (diethyl acetate: petroleum ether 9.5/0.5) or recrystallization in (diethyl ether/ $n$-hexane) gave the pure products in 70 $90 \%$ yields.

2.3. General procedure for the synthesis of $\mathrm{N}, \mathrm{N}$-Bis (2-chloroethyl) sulfamidocarbonyloxymethylphosphonates derivatives (1b-7b)

$N$, N-bis (2-chloroethyl) sulfamidocarbonyloxymethylphosphonates derivatives were obtained in 2 steps: firstly, a solution of $\alpha$ hydroxyphosphonates (1.1 eq) in anhydrous DCM (5 mL) was slowly added dropwise to a stirred solution of chlorosulfonyl isocyanate (CSI) (1 eq, $500.00 \mathrm{mg}, 3.53 \mathrm{mmol}$ ) dissolved in DCM at $0{ }^{\circ} \mathrm{C}$ for $30 \mathrm{~min}$. In a second step, the resulting solution were slowly added to a solution of bis (2-chloroethyl) amine hydrochloride (1 eq, $630.56 \mathrm{mg}, 3.53 \mathrm{mmol}$ ) in anhydrous $\mathrm{DCM}(10 \mathrm{~mL})$ at $0^{\circ} \mathrm{C}$ in the presence of triethylamine (2 eq, $715.02 \mathrm{mg}, 7.07 \mathrm{mmol}$ ), the resulting reaction solution was then stirred at room temperature for $2 \mathrm{~h}$. Finally, the reaction mixture was diluted with dichloromethane $(100 \mathrm{~mL})$, washed with $\mathrm{HCl}(0.1 \mathrm{~N})$ until acidic $\mathrm{pH}$ then extracted with water up to neutral $\mathrm{pH}$. The organic layer was dried over anhydrous sodium sulfate $\left(\mathrm{Na}_{2} \mathrm{SO}_{4}\right)$, filtered and concentrated in vaccuo. The crude product was crystallized in a mixture of diethyl ether/ $n$-hexane (1:1) or purified by column chromatography on silica gel (eluent: $\mathrm{CH}_{2} \mathrm{Cl}_{2}$ : $\mathrm{MeOH}$ (9.8:0.2) to afford the desired $N, N$-bis (2-chloroethyl) sulfamidocarbonyloxymethylphosphonates derivatives (1b-7b) in excellent yields.

(Diethoxyphosphoryl) (phenyl) methyl (N,N-bis(2-chloroethyl) sulfamoyl) carbamate: the product ( $\left.1 \mathrm{~b}, \mathrm{C}_{16} \mathrm{H}_{25} \mathrm{Cl}_{2} \mathrm{~N}_{2} \mathrm{O}_{7} \mathrm{PS}\right)$ was obtained as a colorless oil (160 mg, 92\% yield); $\mathrm{R}_{f} 0.48(\mathrm{DCM} / \mathrm{MeOH})(9.8: 0.2)$; FTIR $(\mathrm{KBr}): 3267(\mathrm{NH}), 1741(\mathrm{CO}), 1366$ and $1155\left(\mathrm{SO}_{2}\right), 1232$ and $1042(\mathrm{PO})$ $\mathrm{cm}^{-1} ;{ }^{31} \mathrm{P}$ NMR (161.9 MHz, $\left.\mathrm{CDCl}_{3}\right) \delta 16.43 \mathrm{ppm} ;{ }^{1} \mathrm{H} \mathrm{NMR}(300 \mathrm{MHz}$, $\left.\mathrm{CDCl}_{3}\right) \delta 1.03\left(\mathrm{t}, \mathrm{J}_{\mathrm{H}-\mathrm{H}} 7.2 \mathrm{~Hz}, 3 \mathrm{H}, \mathrm{CH}_{3}-\mathrm{CH}_{2}\right), 1.32\left(\mathrm{t}, \mathrm{J}_{\mathrm{H}-\mathrm{H}} 7.2 \mathrm{~Hz}, 3 \mathrm{H}, \mathrm{CH}_{3}-\right.$ $\left.\mathrm{CH}_{2}\right)$, 3.51-3.75 (m, 8H, 4Cㅡㅡㄹ), 3.83-3.97 (m, 1H, $\left.\underline{\mathrm{C}}_{2}-\mathrm{CH}_{3}\right), 4.13-4.24(\mathrm{~m}$, $\left.2 \mathrm{H}, \underline{\mathrm{C}}_{2}-\mathrm{CH}_{3}\right), 4.29-4.35\left(\mathrm{~m}, 1 \mathrm{H}, \underline{\mathrm{C}}_{2}-\mathrm{CH}_{3}\right), 5.89$ (d, JH-P $\left.13.8 \mathrm{~Hz}, 1 \mathrm{H},{ }^{*} \mathrm{C} \underline{\mathrm{H}}\right)$,
7.32-7.34 (m, 3H, $\underline{\mathrm{H}}-\mathrm{Ar}), 7.47-7.49$ (m, 2H, $\mathrm{H}-\mathrm{Ar}), 10.62$ (s, $1 \mathrm{H}, \mathrm{NH}$ ) ppm; ${ }^{13} \mathrm{C}$ NMR $\left(100 \mathrm{MHz}, \mathrm{CDCl}_{3}\right) \delta 16.1\left(\mathrm{~d},{ }^{3} J_{C-P} 5.0 \mathrm{~Hz}, \underline{\mathrm{C}} \mathrm{H}_{3}\right), 16.4\left(\mathrm{~d},{ }^{3} J_{C-P}\right.$ $\left.6.0 \mathrm{~Hz}, \underline{\mathrm{CH}}_{3}\right), 41.5\left(2 \underline{\mathrm{CH}}_{2}-\mathrm{Cl}\right), 52.2\left(2 \underline{\mathrm{C}} \mathrm{H}_{2}-\mathrm{N}\right), 63.7\left(\mathrm{~d},{ }^{2} J_{C-P} 7.0 \mathrm{~Hz}, \underline{\mathrm{CH}}_{2^{-}}\right.$ $\left.\mathrm{CH}_{3}\right), 64.1$ (d, $\left.{ }^{2} J_{C-P} 7.0 \mathrm{~Hz}, \underline{\mathrm{CH}}_{2}-\mathrm{CH}_{3}\right), 71.7\left(\mathrm{~d},{ }^{1} \mathrm{~J}_{\mathrm{C}-\mathrm{P}} 174.0 \mathrm{~Hz},{ }^{*} \underline{\mathrm{CH}}\right), 128.2$

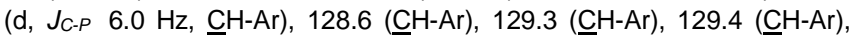
132.4 ( $\underline{\mathrm{C}}-\mathrm{Ar}$ ), 150.6 (d, Jc-p $12.0 \mathrm{~Hz}, \underline{\mathrm{CO}}) \mathrm{ppm} ; \mathrm{MS}\left(\mathrm{ESI}^{+}\right) \mathrm{m} / \mathrm{z}=491.1$ $[\mathrm{M}+\mathrm{H}]^{+} ; \mathrm{HRMS}\left(\mathrm{ESI}^{+}\right): \mathrm{m} / \mathrm{z}[\mathrm{M}+\mathrm{H}]^{+}$calcd for $\mathrm{C}_{16} \mathrm{H}_{25} \mathrm{~N}_{2} \mathrm{O}_{7} \mathrm{PSCl}: 491.0569$; found: 491.0575

(4-Chlorophenyl) (diethoxyphosphoryl) methyl (N,N-bis(2-chloroethyl) sulfamoyl) carbamate: the product $\left(2 \mathrm{~b}, \mathrm{C}_{16} \mathrm{H}_{24} \mathrm{Cl}_{3} \mathrm{~N}_{2} \mathrm{O}_{7} \mathrm{PS}\right)$ was obtained as a white powder (162 mg, $87 \%$ yield); m.p. $138-140{ }^{\circ} \mathrm{C} ; \mathrm{R}_{f} 0.5$ (DCM/MeOH) (9.8:0.2); FTIR (KBr): $3268(\mathrm{NH}), 1741(\mathrm{CO}), 1343$ and $1165\left(\mathrm{SO}_{2}\right), 1260$ and $1024(\mathrm{PO}) \mathrm{cm}^{-1} ;{ }^{31} \mathrm{P} \mathrm{NMR}\left(161.9 \mathrm{MHz}, \mathrm{CDCl}_{3}\right) \delta$ 15.95 ppm; ${ }^{1} \mathrm{H}$ NMR $\left(400 \mathrm{MHz}, \mathrm{CDCl}_{3}\right) \delta 1.13\left(\mathrm{t}, \mathrm{J}_{\mathrm{H}-\mathrm{H}} 6.8 \mathrm{~Hz}, 3 \mathrm{H}, \mathrm{CH}_{3}, \mathrm{CH}_{2}\right)$, $1.36\left(\mathrm{t}, \mathrm{J}_{\mathrm{H}-\mathrm{H}} 6.8 \mathrm{~Hz}, 3 \mathrm{H}, \mathrm{CH}_{3}-\mathrm{CH}_{2}\right), 3.59-3.73\left(\mathrm{~m}, 8 \mathrm{H}, 4 \mathrm{CH}_{2}\right), 3.82-3.86(\mathrm{~m}$, $\left.1 \mathrm{H}, \mathrm{CH}_{2}-\mathrm{CH}_{3}\right), 3.96-4.04\left(\mathrm{~m}, 1 \mathrm{H}, \mathrm{CH}_{2}-\mathrm{CH}_{3}\right), 4.18-4.23\left(\mathrm{~m}, 2 \mathrm{H}, \mathrm{CH}_{2}-\mathrm{CH}_{3}\right)$ $5.90\left(\mathrm{~d}, J_{H-H} 14.0 \mathrm{~Hz}, 1 \mathrm{H},{ }^{*} \mathrm{C} \underline{\mathrm{H}}\right), 7.35\left(\mathrm{~d}, J_{H-H} 8.4 \mathrm{~Hz}, 2 \mathrm{H}, \underline{\mathrm{H}}-\mathrm{Ar}\right), 7.44\left(\mathrm{~d}, J_{H}\right.$ H $2.0 \mathrm{~Hz}, 1 \mathrm{H}, \underline{\mathrm{H}}-\mathrm{Ar}), 7.46$ (d, $\left.J_{H-H} 2.0 \mathrm{~Hz}, 1 \mathrm{H}, \underline{\mathrm{H}}-\mathrm{Ar}\right), 10.22(\mathrm{~s}, 1 \mathrm{H}, \mathrm{NH})$ ppm; ${ }^{13} \mathrm{C}$ NMR (100 MHz, CDCl 3 ) $\delta 16.1$ (d, $\left.{ }^{3} \mathrm{JC}_{\mathrm{C}} 5.0 \mathrm{~Hz}, \mathrm{CH}_{3}\right), 16.4$ (d, $\left.{ }^{3} J_{C-P} 6.0 \mathrm{~Hz}, \mathrm{CH}_{3}\right), 41.5\left(2 \mathrm{CH}_{2}-\mathrm{Cl}\right), 52.2\left(2 \mathrm{CH}_{2}-\mathrm{N}\right), 63.9\left(\mathrm{~d},{ }^{2} J_{C-P} 7.0 \mathrm{~Hz}\right.$ $\left.\underline{\mathrm{CH}}_{2}-\mathrm{CH}_{3}\right), 64.1$ (d, $\left.{ }^{2} \mathrm{~J}_{\mathrm{C}-\mathrm{P}} 7.0 \mathrm{~Hz}, \underline{\mathrm{C}} \mathrm{H}_{2}-\mathrm{CH}_{3}\right), 70.7$ (d, $\left.{ }^{1} \mathrm{~J}_{\mathrm{C}-\mathrm{P}} 174.0 \mathrm{~Hz},{ }^{*} \underline{\mathrm{C}} \mathrm{H}\right)$,

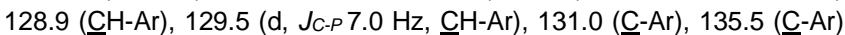
150.4 (d, $\left.J_{C-P} 13.0 \mathrm{~Hz}, \underline{\mathrm{CO}}\right) \mathrm{ppm}$; MS $\left(\mathrm{ESI}^{+}\right) \mathrm{m} / \mathrm{z}=549.0[\mathrm{M}+\mathrm{Na}]^{+}$; HRMS $\left(E S I^{+}\right): \mathrm{m} / \mathrm{z}[\mathrm{M}+\mathrm{Na}]^{+}$calcd for $\mathrm{C}_{16} \mathrm{H}_{24} \mathrm{~N}_{2} \mathrm{O}_{7} \mathrm{NaPSCl}_{3}$ : 547.0004 ; found: 547.0005

Diethoxyphosphoryl) (2-fluorophenyl) methyl (N,N-bis (2-chloroethyl) sulfamoyl)carbamate: the product ( $\left.3 \mathrm{~b}, \mathrm{C}_{16} \mathrm{H}_{24} \mathrm{Cl}_{2} \mathrm{FN}_{2} \mathrm{O}_{7} \mathrm{PS}\right)$ was obtained as a colourless oil $\left(149 \mathrm{mg}, 83 \%\right.$ yield); $\mathrm{R}_{f} 0.52(\mathrm{DCM} / \mathrm{MeOH})(9.8: 0.2)$ FTIR (KBr): $3277(\mathrm{NH}), 1754(\mathrm{CO}), 1369$ and $1157\left(\mathrm{SO}_{2}\right), 1231$ and 1025 (PO) $\mathrm{cm}^{-1} ;{ }^{11} \mathrm{P}$ NMR $\left(121.5 \mathrm{MHz}, \mathrm{CDCl}_{3}\right) \delta 15.77\left(\mathrm{~d}, J_{F-P} 5.7 \mathrm{~Hz}\right) \mathrm{ppm} ;{ }^{1} \mathrm{H}$ NMR (400 MHz, CDCl 3 ) $\delta 1.12$ (t, $\left.J_{H-H} 7.2 \mathrm{~Hz}, 3 \mathrm{H}, \mathrm{CH}_{3}-\mathrm{CH}_{2}\right), 1.38$ (t, JH-H $\left.7.2 \mathrm{~Hz}, 3 \mathrm{H}, \mathrm{C}_{3}-\mathrm{CH}_{2}\right), 3.61-3.73\left(\mathrm{~m}, 8 \mathrm{H}, 4 \mathrm{CH}_{2}\right), 3.86-3.90\left(\mathrm{~m}, 1 \mathrm{H}, \mathrm{CH}_{2}-\right.$ $\left.\mathrm{CH}_{3}\right)$, 3.97-4.03 (m, $\left.1 \mathrm{H}, \mathrm{CH}_{2}-\mathrm{CH}_{3}\right), 4.23-4.27\left(\mathrm{~m}, 2 \mathrm{H}, \mathrm{CH}_{2}-\mathrm{CH}_{3}\right), 6.33(\mathrm{~d}$, $\left.J_{H-H} 14.0 \mathrm{~Hz}, 1 \mathrm{H},{ }^{*} \mathrm{C} \underline{\mathrm{H}}\right), 7.10$ (t, $\left.J_{H-H} 8.4 \mathrm{~Hz}, 1 \mathrm{H}, \underline{\mathrm{H}}-\mathrm{Ar}\right), 7.19$ (t, $J_{H-H} 7.6 \mathrm{~Hz}$ $1 \mathrm{H}, \underline{\mathrm{H}}-\mathrm{Ar}), 7.34-7.41(\mathrm{~m}, 1 \mathrm{H}, \underline{\mathrm{H}}-\mathrm{Ar}), 7.67\left(\mathrm{t}, \mathrm{J}_{\mathrm{H}-\mathrm{H}}=8.2 \mathrm{~Hz}, 1 \mathrm{H}, \underline{\mathrm{H}}-\mathrm{Ar}\right), 10.11$ $(\mathrm{s}, 1 \overline{\mathrm{H}}, \mathrm{NH}) \mathrm{ppm} ;{ }^{13} \mathrm{C} \mathrm{NMR}\left(100 \mathrm{MHz}, \mathrm{CDCl}_{3}\right): \delta=16.2\left(\mathrm{~d},{ }^{3} \mathrm{~J}_{\mathrm{C}-\mathrm{P}} 6.0 \mathrm{~Hz}\right.$, $\left.\underline{\mathrm{CH}}_{3}\right), 16 . \overline{6}\left(\mathrm{~d},{ }^{3} J_{\mathrm{C}-\mathrm{P}} 6.0 \mathrm{~Hz}, \underline{\mathrm{C}} \mathrm{H}_{3}\right), 41.8\left(2 \underline{\mathrm{CH}}_{2}-\mathrm{Cl}\right), 52.5\left(2 \underline{\mathrm{CH}}_{2}-\mathrm{N}\right), 64.1(\mathrm{~d}$,

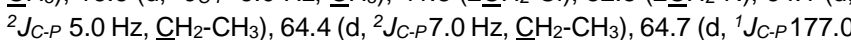

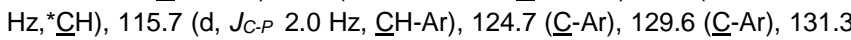
$(\underline{\mathrm{C}}-\mathrm{Ar}), 150.6(\underline{\mathrm{CO}}) \mathrm{ppm} ; \mathrm{MS}\left(\mathrm{ESI}^{+}\right) \mathrm{m} / \mathrm{z}=531.0[\mathrm{M}+\mathrm{Na}]^{+}$; HRMS $\left(\mathrm{ESI}^{+}\right)$: $\mathrm{m} / \mathrm{z}[\mathrm{M}+\mathrm{Na}]^{+}$calcd for $\mathrm{C}_{16} \mathrm{H}_{24} \mathrm{~N}_{2} \mathrm{O}_{7} \mathrm{FNaPSCl}_{2}: 531.0303$; found: 531.0301

(Diethoxyphosphoryl) (4-methoxyphenyl) methyl (N,N-bis(2 chloroethyl) sulfamoyl) carbamate: the product $\left(4 \mathrm{~b}, \mathrm{C}_{17} \mathrm{H}_{27} \mathrm{Cl}_{2} \mathrm{~N}_{2} \mathrm{O}_{8} \mathrm{PS}\right)$ was obtained as a white powder (163 mg, $88 \%$ yield); m.p. $116-118{ }^{\circ} \mathrm{C} ; \mathrm{R}_{f} 0.40$ $(\mathrm{DCM} / \mathrm{MeOH})$ (9.8:0.2); FTIR (KBr): $3260(\mathrm{NH}), 1739(\mathrm{CO}), 1338$ and $1158\left(\mathrm{SO}_{2}\right), 1253$ and $1026(\mathrm{PO}) \mathrm{cm}^{-1} ;{ }^{31} \mathrm{P} \mathrm{NMR}\left(161.9 \mathrm{MHz}, \mathrm{CDCl}_{3}\right) \delta$ $16.67 \mathrm{ppm} ;{ }^{1} \mathrm{H} \mathrm{NMR}\left(300 \mathrm{MHz}, \mathrm{CDCl}_{3}\right) \delta 1.09\left(\mathrm{t}, \mathrm{J}_{\mathrm{H}-\mathrm{H}} 7.2 \mathrm{~Hz}, 3 \mathrm{H}, \mathrm{CH}_{3}-\mathrm{CH}_{2}\right)$ 1.36 (t, J J $\left.\mathrm{H}_{-\mathrm{H}} 7.2 \mathrm{~Hz}, 3 \mathrm{H}, \mathrm{CH}_{3}-\mathrm{CH}_{2}\right), 3.58-3.66\left(\mathrm{~m}, 8 \mathrm{H}, 4 \mathrm{CH}_{2}\right), 3.86-3.78(\mathrm{~m}$, $\left.1 \mathrm{H}, \mathrm{CH}_{2}-\mathrm{CH}_{3}\right), 3.81\left(\mathrm{~s}, 3 \mathrm{H}, \mathrm{OCH}_{3}\right), 3.81-4.00\left(\mathrm{~m}, 1 \mathrm{H}, \mathrm{C}_{2}-\mathrm{CH}_{3}\right), 4.19-4.24$ $\left(\mathrm{m}, 2 \mathrm{H}, \mathrm{CH}_{2}-\mathrm{CH}_{3}\right), 5.88\left(\mathrm{~d}, J_{\mathrm{H}-\mathrm{H}} 13.5 \mathrm{~Hz}, 1 \mathrm{H},{ }^{\star} \mathrm{C} \underline{\mathrm{H}}\right), 6.87$ (d, $J_{H-H} 8.4 \mathrm{~Hz}$, $2 \mathrm{H}, \underline{\mathrm{H}}-\mathrm{Ar}), 7.45\left(\mathrm{~d}, J_{H-H} 1.8 \mathrm{~Hz}, 1 \mathrm{H}, \underline{\mathrm{H}}-\mathrm{Ar}\right), 7.48\left(\mathrm{~d}, J_{H-H} 1.8 \mathrm{~Hz}, 1 \mathrm{H}, \underline{\mathrm{H}}-\right.$ $\mathrm{Ar}), 10.18$ (s, $1 \mathrm{H}, \mathrm{NH}) \mathrm{ppm} ;{ }^{13} \mathrm{C} \mathrm{NMR}\left(100 \mathrm{MHz}, \mathrm{CDCl}_{3}\right) \delta 16.1$ (d, ${ }^{3} J_{C-P}$ $\left.6.0 \mathrm{~Hz}, \underline{\mathrm{CH}}_{3}\right), 16.5\left(\mathrm{~d},{ }^{3} J_{C-P} 5.0 \mathrm{~Hz}, \underline{\mathrm{C}} \mathrm{H}_{3}\right), 41.5\left(2 \underline{\mathrm{CH}}_{2}-\mathrm{Cl}\right), 52.2\left(2 \underline{\mathrm{C}} \mathrm{H}_{2}-\mathrm{N}\right)$, $55.3\left(\mathrm{O}_{\underline{\mathrm{C}}} \mathrm{H}_{3}\right), 63.6\left(\mathrm{~d},{ }^{2} J_{C-p} 7.0 \mathrm{~Hz}, \underline{\mathrm{C}} \mathrm{H}_{2}-\mathrm{CH}_{3}\right), 64.1$ (d, ${ }^{2} J_{C-p} 7.0 \mathrm{~Hz}, \underline{\mathrm{C}} \mathrm{H}_{2}-$

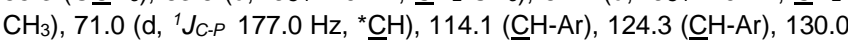
(d, JC-P $6.0 \mathrm{~Hz}, \underline{\mathrm{C}}-\mathrm{Ar}$ ), 130.0 (d, J $J_{C-P} 6.0 \mathrm{~Hz}, \underline{\mathrm{C}}-\mathrm{Ar}$ ), 150.9 (d, $J_{C-P} 13.0 \mathrm{~Hz}$, CO), 160.4 (ㄷ-Ar) ppm; MS $\left(\mathrm{ESI}^{+}\right) \mathrm{m} / \mathrm{z}=543.1[\mathrm{M}+\mathrm{Na}]^{+}$; HRMS $\left(\mathrm{ESI}^{+}\right.$) $\mathrm{m} / \mathrm{z}[\mathrm{M}+\mathrm{Na}]^{+}$calcd for $\mathrm{C}_{17} \mathrm{H}_{27} \mathrm{~N}_{2} \mathrm{O}_{8} \mathrm{NaSCl}_{2} \mathrm{P}: 543.0508$; found: 543.0500

(Diethoxyphosphoryl) (naphthalen-1-yl) methyl (N,N-bis(2-chloroethyl) sulfamoyl) carbamate: the product $\left(5 \mathrm{~b}, \mathrm{C}_{20} \mathrm{H}_{27} \mathrm{Cl}_{2} \mathrm{~N}_{2} \mathrm{O}_{7} \mathrm{PS}\right)$ was obtained as a yellow oil (173 mg, $90 \%$ yield); $\mathrm{R}_{f} 0.45(\mathrm{DCM} / \mathrm{MeOH})(9.8: 0.2)$; FTIR $(\mathrm{KBr}): 3262(\mathrm{NH}), 1746(\mathrm{CO}), 1367$ and $1157\left(\mathrm{SO}_{2}\right), 1230$ and $1042(\mathrm{PO})$ $\mathrm{cm}^{-1}$; ${ }^{31} \mathrm{P}$ NMR (161.9 MHz, $\left.\mathrm{CDCl}_{3}\right) \delta 16.39 \mathrm{ppm}$; ${ }^{1} \mathrm{H}$ NMR $(400 \mathrm{MHz}$, $\left.\mathrm{CDCl}_{3}\right) \delta 1.06\left(\mathrm{t}, \mathrm{J}_{\mathrm{H}-\mathrm{H}} 6.8 \mathrm{~Hz}, 3 \mathrm{H}, \mathrm{CH}_{3}\right), 1.38\left(\mathrm{t}, J_{H-H} 7.2 \mathrm{~Hz}, 3 \mathrm{H}, \mathrm{C}_{3}\right), 3.52-$ 
$3.74\left(\mathrm{~m}, 8 \mathrm{H}, 4\left(\mathrm{CH}_{2}\right)\right), 3.90-4.09\left(\mathrm{~m}, 1 \mathrm{H}, \mathrm{CH}_{2}-\mathrm{CH}_{3}\right), 4.24-4.27\left(\mathrm{~m}, 2 \mathrm{H}, \mathrm{CH}_{2}-\right.$ $\left.\mathrm{CH}_{3}\right)$, 4.33-4.37 (m, $\left.1 \mathrm{H}, \mathrm{C}_{2}-\mathrm{CH}_{3}\right), 6.13\left(\mathrm{~d},{ }^{1} \mathrm{~J}_{\mathrm{H}-\mathrm{P}} 14.0 \mathrm{~Hz}, 1 \mathrm{H}, \mathrm{CH}^{*}\right), 7.48$ 7.54 (m, 2H, $\underline{\mathrm{H}}$-Ar), 7.68-7.70 (m, 1H, $\underline{\mathrm{H}}-\mathrm{Ar}), 7.78-7.85$ (m, 3H, $\mathrm{H}-\mathrm{Ar}), 8.01$ (brs, $1 \mathrm{H}, \underline{\mathrm{H}}-\mathrm{Ar}$ ), 10.41 (s, $1 \mathrm{H}, \mathrm{N} \underline{\mathrm{H}}$ ) ppm; ${ }^{13} \mathrm{C} \mathrm{NMR}\left(100 \mathrm{MHz}, \mathrm{CDCl}_{3}\right) \delta 16.1$ (d, ${ }^{3} \mathrm{JC}_{\mathrm{C}-\mathrm{P}} 5.0 \mathrm{~Hz}, \underline{\mathrm{C}} \mathrm{H}_{3}$ ), 16.5 (d, $\left.{ }^{3} \mathrm{JC}-\mathrm{p} 6.0 \mathrm{~Hz}, \underline{\mathrm{C}} \mathrm{H}_{3}\right), 41.5$ (s, $\left.2 \underline{\mathrm{C}} \mathrm{H}_{2}-\mathrm{Cl}\right), 52.2$ (s, $\left.2 \mathrm{C}_{2}-\mathrm{N}\right), 63.8$ (d, $\left.{ }^{2} J_{C-P} 5.0 \mathrm{~Hz}, \underline{\mathrm{C}}_{2}\right), 64.1$ (d, $\left.{ }^{2} J_{C-P} 7.0 \mathrm{~Hz}, \underline{\mathrm{CH}_{2}}\right), 71.7$ (d, $\left.{ }^{1} J_{C-P} 174.0 \mathrm{~Hz}, \underline{\mathrm{C}}^{*}\right), 125.2$ (d, JC-P $\left.4.0 \mathrm{~Hz}, \underline{\mathrm{CH}}-\mathrm{Ar}\right), 126.6$ ( $\left.\underline{\mathrm{CH}}-\mathrm{Ar}\right), 126.9$ ( $\underline{\mathrm{C}} \mathrm{H}-\mathrm{Ar}), 127.8(\underline{\mathrm{C}} \mathrm{H}-\mathrm{Ar}), 128.0$ (d, Jc-p $8.0 \mathrm{~Hz}, \underline{\mathrm{C}} \mathrm{H}-\mathrm{Ar}), 128.1$ ( $\underline{\mathrm{C}} \mathrm{H}-\mathrm{Ar}), 128.6$

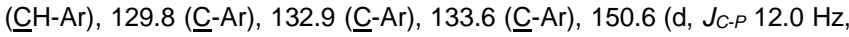
CO) ppm; MS $\left(\mathrm{ESI}^{+}\right) \mathrm{m} / \mathrm{z}=563.1\left[\mathrm{M}+\mathrm{Na}^{+} ; \mathrm{HRMS}(\mathrm{ESI})^{+}\right): \mathrm{m} / \mathrm{z}[\mathrm{M}+\mathrm{Na}]^{+}$ calcd for $\mathrm{C}_{20} \mathrm{H}_{27} \mathrm{~N}_{2} \mathrm{O}_{7} \mathrm{NaSCl}_{2} \mathrm{P}$ : 563.0551 ; found: 563.0551

Diethoxyphosphoryl) (thiophen-2-yl) methyl (N,N-bis(2chloroethyl)sulfamoyl) carbamate: the product (6b, $\left.\mathrm{C}_{14} \mathrm{H}_{23} \mathrm{Cl}_{2} \mathrm{~N}_{2} \mathrm{O}_{7} \mathrm{PS}_{2}\right)$ was obtained as a white powder $\left(141 \mathrm{mg}, 80 \%\right.$ yield); m.p. $130-132{ }^{\circ} \mathrm{C}$; $\mathrm{R}_{f} 0.42(\mathrm{DCM} / \mathrm{MeOH})(9.8: 0.2)$; FTIR $(\mathrm{KBr}): 3280(\mathrm{NH}), 1745(\mathrm{CO}), 1340$ and $1150\left(\mathrm{SO}_{2}\right), 1251$ and $1045(\mathrm{PO}) \mathrm{cm}^{-1} ;{ }^{31} \mathrm{P} \mathrm{NMR}\left(161.9 \mathrm{MHz}, \mathrm{CDCl}_{3}\right)$ $\delta 15.10 \mathrm{ppm} ;{ }^{1} \mathrm{H} \mathrm{NMR}\left(400 \mathrm{MHz}, \mathrm{CDCl}_{3}\right) \delta 1.16\left(\mathrm{t}, \mathrm{J}_{\mathrm{H}-\mathrm{H}} 7.2 \mathrm{~Hz}, 3 \mathrm{H}, \mathrm{CH}_{3}\right)$, 1.35 (t, JH-H $\left.7.2 \mathrm{~Hz}, 3 \mathrm{H}, \mathrm{CH}_{3}\right), 3.64-3.74\left(\mathrm{~m}, 8 \mathrm{H}, \mathrm{CH}_{2}\right), 3.91-3.96(\mathrm{~m}, 1 \mathrm{H}$, $\left.\mathrm{CH}_{2}-\mathrm{CH}_{3}\right), 4.05-4.11\left(\mathrm{~m}, 1 \mathrm{H}, \mathrm{CH}_{2}-\mathrm{CH}_{3}\right), 4.21-4.25\left(\mathrm{~m}, 2 \mathrm{H}, \mathrm{CH}_{2}-\mathrm{CH}_{3}\right), 6.23$ (d, $\left.{ }^{1} \mathrm{~J}_{\mathrm{H}-\mathrm{P}} 14.8 \mathrm{~Hz}, 1 \mathrm{H}, \mathrm{CH}^{*}\right), 7.01-7.03(\mathrm{~m}, 1 \mathrm{H}, \underline{\mathrm{H}}$-vinyl), 7.32 (brs, $1 \mathrm{H}, \underline{\mathrm{H}}-$ vinyl), 7.38 (d, JH-H $4.8 \mathrm{~Hz}, 1 \mathrm{H}, \underline{\mathrm{H}}$-vinyl), $9.58\left(\mathrm{~s}, 1 \mathrm{H}, \mathrm{N}\right.$ ) ppm. ${ }^{13} \mathrm{C} \mathrm{NMR}$ $\left(100 \mathrm{MHz}, \mathrm{CDCl}_{3}\right) \delta 16.1\left(\underline{\mathrm{CH}_{3}}\right), 16.4\left(\underline{\mathrm{CH}}_{3}\right), 41.5\left(2 \mathrm{CH}_{2}-\mathrm{Cl}\right), 52.2\left(2 \mathrm{CH}_{2-}\right.$ $\mathrm{N}), 64.1\left(\mathrm{t},{ }^{2} J_{C-P} 6.0 \mathrm{~Hz}, 2 \underline{\mathrm{C}} \mathrm{H}_{2}\right), 66.2\left(\mathrm{~d},{ }^{1} J_{C-P} 181.0 \mathrm{~Hz}, \underline{\mathrm{C}}^{*}\right), 127.0(\underline{\mathrm{C}} \mathrm{H}-$ vinyl), 128.3 ( $\underline{\mathrm{C}} \mathrm{H}$-vinyl), 129.9 ( $\underline{\mathrm{CH}} \mathrm{H}$-vinyl), 133.4 (ㅁ-vinyl), 150.1 (d, JC-P 9.0

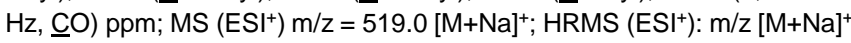
calcd for $\mathrm{C}_{14} \mathrm{H}_{23} \mathrm{~N}_{2} \mathrm{O}_{7} \mathrm{NaPS}_{2} \mathrm{Cl}_{2}$ : 518.9972; found: 518.9959

(Diethoxyphosphoryl) (furan-2-yl) methyl (N,N-bis (2-chloroethyl) sulfamoyl) carbamate: the product $\left(7 \mathrm{~b}, \mathrm{C}_{14} \mathrm{H}_{23} \mathrm{Cl}_{2} \mathrm{~N}_{2} \mathrm{O}_{8} \mathrm{PS}\right)$ was obtained as a brown powder $\left(133 \mathrm{mg}, 78 \%\right.$ yield); m.p. $124-126{ }^{\circ} \mathrm{C} ; \mathrm{R}_{f} 0.40$ (DCM/MeOH) (9.8:0.2); FTIR (KBr): $3285(\mathrm{NH}), 1732(\mathrm{CO}), 1349$ and $1144\left(\mathrm{SO}_{2}\right), 1230$ and $1030(\mathrm{PO}) \mathrm{cm}^{-1} ;{ }^{31} \mathrm{P} \mathrm{NMR}\left(161.9 \mathrm{MHz}, \mathrm{CDCl}_{3}\right) \delta$ 14.12 ppm; ${ }^{1} \mathrm{H}$ NMR $\left(400 \mathrm{MHz}, \mathrm{CDCl}_{3}\right) \delta 1.17$ (t, $\left.\mathrm{JH}_{\mathrm{H}} \mathrm{H} .2 \mathrm{~Hz}, 3 \mathrm{H}, \mathrm{C}_{3}\right)$, $1.31\left(\mathrm{t}, \mathrm{J}_{\mathrm{H}-\mathrm{H}} 7.2 \mathrm{~Hz}, 3 \mathrm{H}, \mathrm{CH}_{3}\right), 3.64-3.73\left(\mathrm{~m}, 8 \mathrm{H}, \mathrm{CH}_{2}\right), 3.96-4.06(\mathrm{~m}, 1 \mathrm{H}$, $\left.\mathrm{CH}_{2}-\mathrm{CH}_{3}\right), 4.08-4.18\left(\mathrm{~m}, 1 \mathrm{H}, \mathrm{CH}_{2}-\mathrm{CH}_{3}\right), 4.20-4.24\left(\mathrm{~m}, 2 \mathrm{H}, \mathrm{CH}_{2}-\mathrm{CH}_{3}\right), 6.06$ (d, $\left.{ }^{\top} \mathrm{J}_{\mathrm{H}-\mathrm{P}} 16.0 \mathrm{~Hz}, 1 \mathrm{H}, \mathrm{CH}^{*}\right), 6.36-6.37$ (m, $1 \mathrm{H}, \underline{\mathrm{H}}$-vinyl), $6.62\left(\mathrm{t}, \mathrm{J}_{\mathrm{H}-\mathrm{H}} 2.4 \mathrm{~Hz}\right.$, $1 \mathrm{H}, \underline{\mathrm{H}}$-vinyl), 7.42-7.43 (m, $1 \mathrm{H}, \underline{\mathrm{H}}$-vinyl), $10.23(\mathrm{~s}, 1 \mathrm{H}, \mathrm{NH}) \mathrm{ppm} ;{ }^{13} \mathrm{C} \mathrm{NMR}$ $\left(100 \mathrm{MHz}, \mathrm{CDCl}_{3}\right) \delta 16.1$ (d, $\left.{ }^{3} \mathrm{JC}_{\mathrm{P}} \mathrm{P} 6.0 \mathrm{~Hz}, \underline{\mathrm{CH}}_{3}\right), 16.4\left(\mathrm{~d},{ }^{3} \mathrm{JC}-\mathrm{p} 6.0 \mathrm{~Hz}, \mathrm{CH}_{3}\right)$, $41.6\left(\mathrm{~s}, 2 \underline{\mathrm{C}} \mathrm{H}_{2}-\mathrm{Cl}\right), 52.3\left(\mathrm{~s}, 2 \underline{\mathrm{C}} \mathrm{H}_{2}-\mathrm{N}\right), 64.1\left(\mathrm{t},{ }^{2} J_{C-P} 8.0 \mathrm{~Hz}, 2 \underline{\mathrm{CH}}_{2}\right), 66.3(\mathrm{~d}$, $\left.{ }^{1} J_{C-p} 189.0 \mathrm{~Hz}, \underline{\mathrm{C}}^{*}\right), 111.0\left(\underline{\mathrm{C}} \mathrm{H}\right.$-vinyl), $112.7\left(\mathrm{~d}, J_{C-p} 5.0 \mathrm{~Hz}, \underline{\mathrm{C}} \mathrm{H}\right.$-vinyl), 144.1

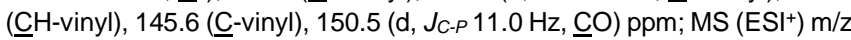
$=503.0 \quad[\mathrm{M}+\mathrm{Na}]^{+} ;$HRMS $\left(\mathrm{ESI}^{+}\right): \mathrm{m} / \mathrm{z} \quad[\mathrm{M}+\mathrm{Na}]^{+}$calcd for $\mathrm{C}_{14} \mathrm{H}_{23} \mathrm{~N}_{2} \mathrm{O}_{8} \mathrm{NaPSCl}_{2}$ : 503.0190; found: 503.0187

\subsection{General procedure for the synthesis of cyclic compounds (1c-7c)}

$N, \mathrm{~N}$-bis (2-chloroethyl) sulfamidocarbonyloxyphosphonates $\mathbf{1 b}(100.00$ $\mathrm{mg}, 203.53 \mu \mathrm{mol})$ was dissolved in dry acetonitrile $(20 \mathrm{~mL})$ at room temperature then we added $\mathrm{K}_{2} \mathrm{CO}_{3}(23.13 \mathrm{mg}, 203.53 \mu \mathrm{mol})$. The mixture was heated to reflux under stirring for $2 \mathrm{~h}$. The reaction mixture was evaporated, diluted with ethyl acetate $(30 \mathrm{~mL})$ and then extracted with water. The organic layer was combined and dried over anhydrous sodium sulfate $\left(\mathrm{Na}_{2} \mathrm{SO}_{4}\right)$, filtered and concentrated in vacuo. All products (1c-7c) were obtained pure without further treatment with a total transformation of the starting material.

(Diethoxyphosphoryl) (phenyl) methyl 5-(2-chloroethyl)-1,2,5thiadiazolidine-2-carboxylate 1,1-dioxide: the product (1c, $\mathrm{C}_{16} \mathrm{H}_{24} \mathrm{ClN}_{2} \mathrm{O}_{7} \mathrm{PS}$ ) was obtained as a white powder $(92,58 \mathrm{mg}, 100 \%$ yield); m.p $110-112{ }^{\circ} \mathrm{C} ; \mathrm{R}_{f} 0.60(\mathrm{DCM} / \mathrm{MeOH})(9.8: 0.2)$; FTIR (KBr): 1735 (CO), 1357 and $1152\left(\mathrm{SO}_{2}\right), 1260$ and $1021(\mathrm{PO}) \mathrm{cm}^{-1} ;{ }^{31} \mathrm{P} \mathrm{NMR}(161.9 \mathrm{MHz}$, $\left.\mathrm{CDCl}_{3}\right) \delta 15.05 \mathrm{ppm}$; ${ }^{1} \mathrm{H}$ NMR $\left(400 \mathrm{MHz}, \mathrm{CDCl}_{3}\right) \delta 1.22-1.29(\mathrm{~m}, 6 \mathrm{H}$, $\left.2 \mathrm{CH}_{3}\right), 3.46-3.49\left(\mathrm{~m}, 2 \mathrm{H},\left(\mathrm{CH}_{2}\right)_{c y c}-\mathrm{NCH}_{2}\right), 3.56\left(\mathrm{t}, \mathrm{JH}_{\mathrm{H}-\mathrm{H}}=6.8 \mathrm{~Hz}, 2 \mathrm{H}, \mathrm{CH}_{2}-\right.$ $\left.\mathrm{CH}_{2}-\mathrm{Cl}\right), 3.73\left(\mathrm{t}, J_{H-H}=6.8 \mathrm{~Hz},\left(\mathrm{CH}_{2}\right)_{\mathrm{cyc}}-\mathrm{NCO}\right), 3.92\left(\mathrm{t}, J_{H-H}=6.8 \mathrm{~Hz}, \overline{2 \mathrm{H}}\right.$,

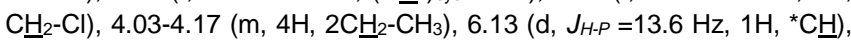
7.32-7.39 (m, 3H, $\mathrm{H}-\mathrm{Ar}), 7.50-7.53$ (m, $2 \mathrm{H}, \mathrm{H}-\mathrm{Ar}) \mathrm{ppm} ;{ }^{13} \mathrm{C} \mathrm{NMR}(100 \mathrm{MHz}$, $\left.\mathrm{CDCl}_{3}\right) \delta 16.3\left(\mathrm{~d},{ }^{3} \mathrm{~J}_{C-P}=2.0 \mathrm{~Hz}, \underline{\mathrm{CH}}_{3}\right), 16.4\left(\mathrm{~d},{ }^{3} \mathrm{JC}_{\mathrm{P}}=3.0 \mathrm{~Hz}, \underline{\mathrm{C}} \mathrm{H}_{3}\right), 41.6$
$\left.\left.\left(\mathrm{CH}_{2}\right)_{\text {cyc }}-\mathrm{NCO}\right), 43.4\left(\mathrm{CH}_{2}-\mathrm{Cl}\right), 45.2\left(\mathrm{CH}_{2}-\mathrm{CH}_{2}-\mathrm{Cl}\right), 48.8\left(\mathrm{CH}_{2}\right)_{\mathrm{cyc}}-\mathrm{NCH}_{2}\right)$, $63.8\left(\mathrm{~d},{ }^{2} J_{C-P}=6.0 \mathrm{~Hz}, 2 \underline{\mathrm{C}} \mathrm{H}_{2}-\mathrm{CH}_{3}\right), 73.0\left(\mathrm{~d},{ }^{1} J_{C-P}=168.0 \mathrm{~Hz},{ }^{*} \underline{\mathrm{C}} \mathrm{H}\right), 127.5$ (d, $\left.J_{C-P}=5.0 \mathrm{~Hz}, \underline{\mathrm{CH}}-\mathrm{Ar}\right), 128.5\left(\mathrm{~d}, J_{C-P}=2.0 \mathrm{~Hz}, \underline{\mathrm{C}} \mathrm{H}-\mathrm{Ar}\right), 128.8\left(\mathrm{~d}, J_{C-P}=\right.$

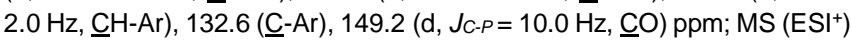
$\mathrm{m} / \mathrm{z}=455.1[\mathrm{M}+\mathrm{H}]^{+} ; \mathrm{HRMS}\left(\mathrm{ESI}^{+}\right): \mathrm{m} / \mathrm{z}[\mathrm{M}+\mathrm{H}]^{+}$calcd for $\mathrm{C}_{16} \mathrm{H}_{25} \mathrm{~N}_{2} \mathrm{O} 7 \mathrm{PSCl}:$ 455.0808; found: 455.0809

(4-chlorophenyl)(diethoxyphosphoryl) methyl 5-(2-chloroethyl)-1,2,5thiadiazolidine-2-carboxylate 1,1-dioxide: the product (2c, $\left.\mathrm{C}_{16} \mathrm{H}_{23} \mathrm{Cl}_{2} \mathrm{~N}_{2} \mathrm{O}_{7} \mathrm{PS}\right)$ was obtained as an colorless oil $(93,07 \mathrm{mg}, 100 \%$ yield); $\mathrm{R}_{f} 0.68(\mathrm{DCM} / \mathrm{MeOH})(9.8: 0.2)$; FTIR (KBr): 1749 (CO), 1361 and $1156\left(\mathrm{SO}_{2}\right), 1229$ and $1018(\mathrm{PO}) \mathrm{cm}^{-1} ;{ }^{31} \mathrm{P} \mathrm{NMR}\left(161.9 \mathrm{MHz}, \mathrm{CDCl}_{3}\right) \delta$ $14.51 \mathrm{ppm} ;{ }^{1} \mathrm{H}$ NMR $\left(400 \mathrm{MHz}, \mathrm{CDCl}_{3}\right) \delta 1.25-1.29\left(\mathrm{~m}, 6 \mathrm{H}, 2 \mathrm{C}_{3}\right), 3.45$ $3.48\left(\mathrm{td}, J_{1}=4.0 \mathrm{~Hz}, J_{2}=6.8 \mathrm{~Hz}, 2 \mathrm{H},\left(\mathrm{CH}_{2}\right) \mathrm{cyc}_{-}-\mathrm{NCH}_{2}\right), 3.56\left(\mathrm{t}, J_{H-H}=6.4 \mathrm{~Hz}\right.$ $\left.2 \mathrm{H}, \mathrm{CH}_{2}-\mathrm{CH}_{2}-\mathrm{Cl}\right), 3.71$ (t, JH-H $\left.=6.4 \mathrm{~Hz},\left(\mathrm{CH}_{2}\right)_{\mathrm{cyc}}-\mathrm{NCO}\right), 3.90$ (t, JH-H $=6.8$ $\left.\mathrm{Hz}, 2 \mathrm{H}, \mathrm{C}_{2}-\mathrm{Cl}\right), 4.04-4.19\left(\mathrm{~m}, 4 \mathrm{H}, 2 \mathrm{C}_{2}-\mathrm{CH}_{3}\right), 6.07$ (d, JH-P $=13.6 \mathrm{~Hz}, 1 \mathrm{H}$, $\left.{ }^{*} \mathrm{CH}\right), 7.32\left(\mathrm{~d}, \mathrm{~J}_{\mathrm{H}-\mathrm{H}}=8.4 \mathrm{~Hz}, 2 \mathrm{H}, \underline{\mathrm{H}}-\mathrm{Ar}\right), 7.43\left(\mathrm{~d}, J_{H-H}=2.0 \mathrm{~Hz}, 1 \mathrm{H}, \underline{\mathrm{H}}-\mathrm{Ar}\right)$, $7.46\left(\mathrm{~d},, J_{H-H}=2.0 \mathrm{~Hz}, 1 \mathrm{H}, \underline{\mathrm{H}}-\mathrm{Ar}\right) \mathrm{ppm} ;{ }^{13} \mathrm{C} \mathrm{NMR}\left(100 \mathrm{MHz}, \mathrm{CDCl}_{3}\right) \delta 16.3$ $\left.\left(\mathrm{d},{ }^{3} \mathrm{~J}_{\mathrm{C}-\mathrm{P}}=2.0 \mathrm{~Hz}, \mathrm{CH}_{3}\right), 16.4\left(\mathrm{~d},{ }^{3} \mathrm{~J}_{\mathrm{C}-\mathrm{P}}=3.0 \mathrm{~Hz}, \mathrm{CH}_{3}\right), 41.5\left(\mathrm{CH}_{2}\right)_{\mathrm{cyc}}-\mathrm{NCO}\right)$, $43.5\left(\mathrm{CH}_{2}-\mathrm{Cl}\right), 45.1\left(\mathrm{CH}_{2}-\mathrm{CH}_{2}-\mathrm{Cl}\right), 48.8\left(\mathrm{CH}_{2}\right)$ cyc- $\left.\mathrm{NCH}_{2}\right), 63.9\left(\mathrm{~d},{ }^{2} \mathrm{JC}-\mathrm{p}=6.0\right.$ $\left.\mathrm{Hz}, 2{ }^{2} \mathrm{CH}_{2}-\mathrm{CH}_{3}\right), 72.3\left(\mathrm{~d},{ }^{1} \mathrm{~J}_{\mathrm{C}-\mathrm{P}}=168.0 \mathrm{~Hz},{ }^{*} \mathrm{CH}\right), 128.5\left(\mathrm{~d}, J_{\mathrm{C}-\mathrm{P}}=2.0 \mathrm{~Hz}\right.$ $\underline{\mathrm{C}} \mathrm{H}-\mathrm{Ar}), 128.9\left(\mathrm{~d}, J_{\mathrm{C}-P}=5.0 \mathrm{~Hz}, \underline{\mathrm{C}} \mathrm{H}-\mathrm{Ar}\right), 131.3\left(\mathrm{~d}, J_{C-P}=2.0 \mathrm{~Hz}, \underline{\mathrm{C}}-\mathrm{Ar}\right)$, $134.9\left(\mathrm{~d}, J_{C-P}=3.0 \mathrm{~Hz}, \mathrm{C}-\mathrm{Ar}\right), 149.1$ (d, $\left.J_{C-P}=11.0 \mathrm{~Hz}, \mathrm{CO}\right) \mathrm{ppm} ; \mathrm{MS}\left(\mathrm{ESI}{ }^{+}\right.$ $\mathrm{m} / \mathrm{z}=511.0 \quad[\mathrm{M}+\mathrm{Na}]^{+} ;$HRMS $\left(E S l^{+}\right): \mathrm{m} / \mathrm{z} \quad[\mathrm{M}+\mathrm{Na}]^{+}$calcd for $\mathrm{C}_{16} \mathrm{H}_{23} \mathrm{~N}_{2} \mathrm{O}_{7} \mathrm{NaPSCl}_{2}$ : 511.0238; found: 511.0238

(Diethoxyphosphoryl)(2-fluorophenyl) methyl 5-(2-chloroethyl)-1,2,5thiadiazolidine-2-carboxylate 1,1-dioxide: the product (3c $\left.\mathrm{C}_{16} \mathrm{H}_{23} \mathrm{ClFN}_{2} \mathrm{O}_{7} \mathrm{PS}\right)$ was obtained as a white crystal $(92,84 \mathrm{mg}, 100 \%$ yield); m.p. $106-108^{\circ} \mathrm{C} ; \mathrm{R}_{f} 0.70(\mathrm{DCM} / \mathrm{MeOH})$ (9.8 :0.2); FTIR (KBr): 1740 (CO), 1323 and $1157\left(\mathrm{SO}_{2}\right), 1258$ and 1025 (PO) cm ${ }^{-1} ;{ }^{31} \mathrm{P}$ NMR $(121.48$ $\left.\mathrm{MHz}, \mathrm{CDCl}_{3}\right) \delta 14.43\left(\mathrm{~d}, \mathrm{~J}_{F-P}=4.6 \mathrm{~Hz}\right) \mathrm{ppm} ;{ }^{1} \mathrm{H} \mathrm{NMR}\left(400 \mathrm{MHz}, \mathrm{CDCl}_{3}\right) \delta$ $1.26\left(\mathrm{t}, J_{H-H}=7.2 \mathrm{~Hz}, 6 \mathrm{H}, 2 \mathrm{CH}_{3}\right), 3.45-3.49\left(\mathrm{td}, J_{1}=3.6 \mathrm{~Hz}, J_{2}=6.4 \mathrm{~Hz}, 2 \mathrm{H}\right.$ $\left(\mathrm{CH}_{2}\right)$ cyc- $\mathrm{NCH}_{2}$ ), $3.56\left(\mathrm{t}, J_{H-H}=6.4 \mathrm{~Hz}, 2 \mathrm{H}, \mathrm{CH}_{2}-\mathrm{CH}_{2}-\mathrm{Cl}\right), 3.72\left(\mathrm{t}, J_{H-H}=6.4\right.$ $\left.\mathrm{Hz},\left(\mathrm{CH}_{2}\right)_{\mathrm{cyc}}-\mathrm{NCO}\right), 3.91\left(\mathrm{t}, \mathrm{JH}_{\mathrm{H}} \mathrm{H}=6.4 \mathrm{~Hz}, 2 \mathrm{H}, \mathrm{CH}_{2}-\mathrm{Cl}\right), 4.10-4.21(\mathrm{~m}, 4 \mathrm{H}$, $\left.2 \mathrm{CH}_{2}-\mathrm{CH}_{3}\right), 6.46\left(\mathrm{~d}, J_{H-P}=13.2 \mathrm{~Hz}, 1 \mathrm{H},{ }^{*} \mathrm{CH}\right), 7.05\left(\mathrm{t}, J_{H-H}=9.6 \mathrm{~Hz}, 2 \mathrm{H}\right.$, $\underline{\mathrm{H}}-\mathrm{Ar}), 7.18\left(\mathrm{t}, \mathrm{J}_{\mathrm{H}-\mathrm{H}}=7.6 \mathrm{~Hz}, 2 \mathrm{H}, \underline{\mathrm{H}}-\mathrm{Ar}\right), 7.29-7.33(\mathrm{~m}, 1 \mathrm{H}, \underline{\mathrm{H}}-\mathrm{Ar}), 7.63(\mathrm{br}$ $\left.\mathrm{t}, J_{\mathrm{H}-\mathrm{H}}=7.6 \mathrm{~Hz}, 1 \mathrm{H}, \mathrm{H}-\mathrm{Ar}\right) \mathrm{ppm} ;{ }^{13} \mathrm{C}$ NMR $\left(100 \mathrm{MHz}, \mathrm{CDCl}_{3}\right) \delta 16.3\left(\mathrm{~d},{ }^{3} \mathrm{~J} \mathrm{C}\right.$ $\left.\left.P=5.0 \mathrm{~Hz}, 2 \underline{\mathrm{CH}_{3}}\right), 41.6\left(\underline{\mathrm{C}} \mathrm{H}_{2}\right)_{\mathrm{cyc}}-\mathrm{NCO}\right), 43.4\left(\underline{\mathrm{CH}}_{2}-\mathrm{Cl}\right), 45.2\left(\mathrm{C}_{2}-\mathrm{CH}_{2}-\mathrm{Cl}\right)$, $\left.48.8\left(\mathrm{CH}_{2}\right)_{\mathrm{cyc}}-\mathrm{NCH}_{2}\right), 63.8\left(\mathrm{~d},{ }^{2} \mathrm{~J} \mathrm{C}-\mathrm{P}=7.0 \mathrm{~Hz}, \underline{\mathrm{CH}}_{2}-\mathrm{CH}_{3}\right), 63.9\left(\mathrm{~d},{ }^{2} \mathrm{~J}_{C-p}=7.0\right.$

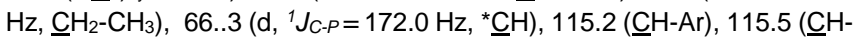

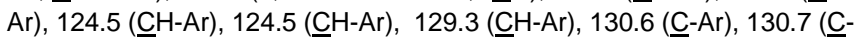
Ar), 149.0 (드) ppm; MS (ESI+) m/z = 495.1 [M+Na] $]^{+}$HRMS $\left(\mathrm{ESI}^{+}\right): \mathrm{m} / \mathrm{z}$ $[\mathrm{M}+\mathrm{Na}]^{+}$calcd for $\mathrm{C}_{16} \mathrm{H}_{23} \mathrm{~N}_{2} \mathrm{O}_{7} \mathrm{FNaPSCl}: 495.0533$; found: 495.0534

(Diethoxyphosphoryl)(4-methoxyphenyl) methyl 5-(2-chloroethyl)-1,2,5thiadiazolidine-2-carboxylate 1,1-dioxide: the product (4c $\left.\mathrm{C}_{17} \mathrm{H}_{26} \mathrm{CIN}_{2} \mathrm{O}_{8} \mathrm{PS}\right)$ was obtained as an colorless oil $(93,01 \mathrm{mg}, 100 \%$ yield); $\mathrm{R}_{f} 0.65$ (DCM/MeOH) (9.8:0.2); FTIR (KBr): 1740 (CO), 1323 and 1157 (SO $), 1258$ and $1025(\mathrm{PO}) \mathrm{cm}^{-1}$; ${ }^{31} \mathrm{P}$ NMR $\left(161.9 \mathrm{MHz}, \mathrm{CDCl}_{3}\right) \delta 15.44$ ppm; ${ }^{1} \mathrm{H}$ NMR $\left(400 \mathrm{MHz}, \mathrm{CDCl}_{3}\right) \delta 1.25-1.30\left(\mathrm{td}, J_{1}=2.4 \mathrm{~Hz}, J_{2}=6.8 \mathrm{~Hz}\right.$, $\left.6 \mathrm{H}, 2 \mathrm{C}_{3}\right), 3.46-3.50$ (td, $J_{1}=1.6 \mathrm{~Hz}, J_{2}=6.4 \mathrm{~Hz},\left(\mathrm{CH}_{2}\right)$ cyc $\left._{2}-\mathrm{NCH}_{2}\right), 3.56$ (t, $\left.J_{H-H}=6.8 \mathrm{~Hz}, 2 \mathrm{H}, \mathrm{CH}_{2}-\mathrm{CH}_{2}-\mathrm{Cl}\right), 3.73\left(\mathrm{t}, J_{H-H}=6.4 \mathrm{~Hz},\left(\mathrm{CH}_{2}\right)_{\mathrm{cyc}}-\mathrm{NCO}\right), 3.81$ $\left(\mathrm{s}, 3 \mathrm{H}, \mathrm{OCH}_{3}\right), 3.92\left(\mathrm{t}, \mathrm{J}_{\mathrm{H}-\mathrm{H}}=6.4 \mathrm{~Hz}, 2 \mathrm{H}, \mathrm{CH}_{2}-\mathrm{Cl}\right), 4.07-4.17\left(\mathrm{~m}, 4 \mathrm{H}, 2 \mathrm{C}_{2}-\right.$ $\left.\mathrm{CH}_{3}\right), 6.07\left(\mathrm{~d}, J_{H-P}=12.8 \mathrm{~Hz}, 1 \mathrm{H},{ }^{*} \mathrm{CH}\right), 6.89\left(\mathrm{~d}, J_{H-H}=8.8 \mathrm{~Hz}, 2 \mathrm{H}, \underline{\mathrm{H}}-\overline{\mathrm{Ar}}\right)$, 7. $44\left(\mathrm{~d}, J_{H-H}=2.0 \mathrm{OHz}, 1 \mathrm{H}, \underline{\mathrm{H}}-\mathrm{Ar}\right), 7.47\left(\mathrm{~d}, J_{H-H}=1.6 \mathrm{~Hz}, 1 \mathrm{H}, \underline{\mathrm{H}}-\mathrm{Ar}\right) \mathrm{ppm}$

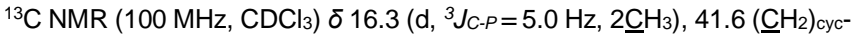
$\left.\mathrm{NCO}), 43.4\left(\underline{\mathrm{CH}}_{2}-\mathrm{Cl}\right), 45.2\left(\underline{\mathrm{CH}}_{2}-\mathrm{CH}_{2}-\mathrm{Cl}\right), 48.8\left(\mathrm{CH}_{2}\right)_{\mathrm{cyc}}-\mathrm{NCH}_{2}\right), 55.3$

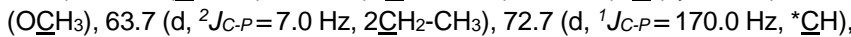
114.1 ( $\underline{\mathrm{C}} \mathrm{H}-\mathrm{Ar}), 124.6$ ( $\underline{\mathrm{C}} \mathrm{H}-\mathrm{Ar}), 129.3\left(\mathrm{~d}, J_{C-P}=5.0 \mathrm{~Hz}, \underline{\mathrm{C}}-\mathrm{Ar}\right), 149.2(\mathrm{~d}, J \mathrm{C}$ $P=11.0 \mathrm{~Hz}, \underline{\mathrm{CO}}), 160.1(\underline{\mathrm{C}}-\mathrm{Ar}) \mathrm{ppm} ; \mathrm{MS}\left(\mathrm{ESI}^{+}\right) \mathrm{m} / \mathrm{z}=507.1[\mathrm{M}+\mathrm{Na}]^{+}$; HRMS $\left(\mathrm{ESI}^{+}\right): \mathrm{m} / \mathrm{z}[\mathrm{M}+\mathrm{Na}]^{+}$calcd for $\mathrm{C}_{17} \mathrm{H}_{26} \mathrm{~N}_{2} \mathrm{O}_{8} \mathrm{NaSCIP}: 507.0734$ found: 507.0734

(Diethoxyphosphoryl) (naphthalen-1-yl) methyl 5-(2-chloroethyl)-1,2,5thiadiazolidine-2-carboxylate 1,1-dioxide: the product $(5 \mathrm{c}$, $\mathrm{C}_{20} \mathrm{H}_{26} \mathrm{ClN}_{2} \mathrm{O}_{7} \mathrm{PS}$ ) was obtained as a white powder ( $93,27 \mathrm{mg}, 100 \%$ yield); m.p. $150-152^{\circ} \mathrm{C} ; \mathrm{R}_{f} 0.63(\mathrm{DCM} / \mathrm{MeOH})(9.8: 0.2)$; FTIR (KBr): 1734 (CO), 
1330 and $1165\left(\mathrm{SO}_{2}\right), 1264$ and $1017(\mathrm{PO}) \mathrm{cm}^{-1} ;{ }^{31} \mathrm{P} \mathrm{NMR}(161.9 \mathrm{MHz}$, $\left.\mathrm{CDCl}_{3}\right) \delta 15.01 \mathrm{ppm} ;{ }^{1} \mathrm{H} \mathrm{NMR}\left(400 \mathrm{MHz}, \mathrm{CDCl}_{3}\right) \delta 1.24\left(\mathrm{t}, J_{\mathrm{H}-\mathrm{H}}=7.2 \mathrm{~Hz}\right.$, $\left.3 \mathrm{H}, \mathrm{CH}_{3}\right), 1.28\left(\mathrm{t}, \mathrm{J}_{\mathrm{H}-\mathrm{H}}=7.2 \mathrm{~Hz}, 3 \mathrm{H}, \mathrm{CH}_{3}\right), 3.49\left(\mathrm{t}, \mathrm{J}_{\mathrm{H}-\mathrm{H}}=6.4 \mathrm{~Hz}, 2 \mathrm{H}\right.$, $\left.\left(\mathrm{CH}_{2}\right)_{\mathrm{cyc}}-\mathrm{NCH}_{2}\right), 3.56\left(\mathrm{t}, J_{H-H}=6.4 \mathrm{~Hz}, 2 \mathrm{H}, \mathrm{CH}_{2}-\mathrm{CH}_{2}-\mathrm{Cl}\right), 3.73\left(\mathrm{t}, J_{H-H}=6.4\right.$ $\mathrm{Hz},\left(\mathrm{C}_{2}\right)_{\text {cyc }}-\mathrm{NCO}$ ), 3.91-3.94 (td, $J_{1}=1.6 \mathrm{~Hz}, J_{2}=6.4 \mathrm{~Hz}, 2 \mathrm{H}, \mathrm{CH}_{2}-\mathrm{Cl}$ ),

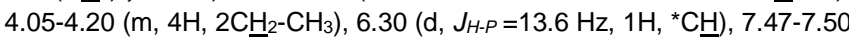
(m, 2H, H-Ar), 7.63 (brd, $\left.J_{H-H}=8.4 \mathrm{~Hz}, 1 \mathrm{H}, \quad \mathrm{H}-\mathrm{Ar}\right) 7.81-7.86(\mathrm{~m}, 3 \mathrm{H}, \mathrm{H}-$ $\mathrm{Ar}), 8.01$ (brs, $1 \mathrm{H}, \underline{\mathrm{H}}-\mathrm{Ar}) \mathrm{ppm} ;{ }^{13} \mathrm{C} \mathrm{NMR}\left(100 \overline{\mathrm{MHz}}, \mathrm{CDCl}_{3}\right) \delta 16.3\left(\mathrm{~d},{ }^{3} \mathrm{JC}\right.$ $\left.P=2.0 \mathrm{~Hz}, \underline{\mathrm{CH}_{3}}\right), 16.4$ (d, $\left.\left.{ }^{3} \mathrm{~J}-\mathrm{P}=3.0 \mathrm{~Hz}, \underline{\mathrm{CH}}_{3}\right), 41.6\left(\underline{\mathrm{CH}}_{2}\right)_{\text {cyc }}-\mathrm{NCO}\right), 43.3$

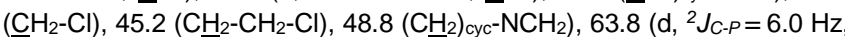
$\left.2 \underline{\mathrm{C}} \mathrm{H}_{2}-\mathrm{CH}_{3}\right), 73.2\left(\mathrm{~d},{ }^{1} \mathrm{~J}_{\mathrm{C}-\mathrm{P}}=168.0 \mathrm{~Hz},{ }^{*} \underline{\mathrm{C}} \mathrm{H}\right), 124.8\left(\mathrm{~d}, J_{\mathrm{C}-\mathrm{P}}=4.0 \mathrm{~Hz}, \underline{\mathrm{CH}}-\right.$

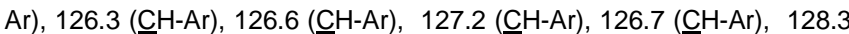

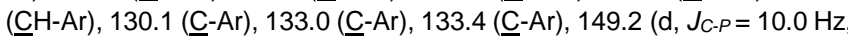
CO) $\mathrm{ppm} ; \mathrm{MS}\left(\mathrm{ESI}^{+}\right) \mathrm{m} / \mathrm{z}=527.1[\mathrm{M}+\mathrm{Na}]^{+} ; \mathrm{HRMS}\left(\mathrm{ESI}^{+}\right): \mathrm{m} / \mathrm{z}[\mathrm{M}+\mathrm{Na}]^{+}$ calcd for $\mathrm{C}_{20} \mathrm{H}_{26} \mathrm{~N}_{2} \mathrm{O}_{7} \mathrm{NaSCIP:}$ 527.0788; found: 527.0785

(Diethoxyphosphoryl) (thiophen-2-yl) methyl 5-(2-chloroethyl)-1,2,5thiadiazolidine-2-carboxylate 1,1-dioxide: : the product (6c, $\left.\mathrm{C}_{14} \mathrm{H}_{22} \mathrm{ClN}_{2} \mathrm{O}_{7} \mathrm{PS}_{2}\right)$ was obtained as an colorless oil $(92,67 \mathrm{mg}, 100 \%$ yield); $\mathrm{R}_{f} 0.50$ (DCM/MeOH) (9.8:0.2); FTIR (KBr): $1744(\mathrm{CO}), 1366$ and $1155\left(\mathrm{SO}_{2}\right), 1224$ and $1018(\mathrm{PO}) \mathrm{cm}^{-1}$; ${ }^{11} \mathrm{P} \mathrm{NMR}\left(161.9 \mathrm{MHz}, \mathrm{CDCl}_{3}\right) \delta$ $13.89 \mathrm{ppm} ;{ }^{1} \mathrm{H}$ NMR $\left(400 \mathrm{MHz}, \mathrm{CDCl}_{3}\right) \delta 1.24\left(\mathrm{~m}, 6 \mathrm{H}, 2 \mathrm{CH}_{3}\right), 3.46$ (t, JH-H $\left.=6.4 \mathrm{~Hz}, 2 \mathrm{H},\left(\mathrm{CH}_{2}\right)_{\mathrm{cyc}}-\mathrm{NCH}_{2}\right), 3.55\left(\mathrm{t}, \mathrm{J}_{\mathrm{H}-\mathrm{H}}=6.4 \mathrm{~Hz}, 2 \mathrm{H}, \mathrm{CH}_{2}-\mathrm{CH}_{2}-\mathrm{Cl}\right), 3.70$ (t, $\left.J_{H-H}=6.4 \mathrm{~Hz},\left(\mathrm{CH}_{2}\right)_{\mathrm{cyc}}-\mathrm{NCO}\right), 3.91\left(\mathrm{t}, J_{\mathrm{H}-\mathrm{H}}=6.8 \mathrm{~Hz}, 2 \mathrm{H}, \mathrm{C}_{2}-\mathrm{Cl}\right), 4.14-$ $4.20\left(\mathrm{~m}, 4 \mathrm{H}, 2 \mathrm{CH}_{2}-\mathrm{CH}_{3}\right), 6.36$ (d, $J_{H-P}=13.6 \mathrm{~Hz}, 1 \mathrm{H},{ }^{*} \mathrm{CH}$ ), 6.99-7.01 (dd, $\left.J_{1}=3.6 \mathrm{~Hz}, J_{2}=5.2 \mathrm{~Hz}, 1 \mathrm{H}, \underline{\mathrm{H}}-\mathrm{Ar}\right), 7.29-7.30(\mathrm{~m}, 1 \mathrm{H}, \underline{\mathrm{H}}-\mathbf{A r}), 7.34-7.36(2 \mathrm{t}$, $\left.J_{1}=1.2 \mathrm{~Hz}, J_{2}=5.2 \mathrm{~Hz}, 1 \mathrm{H}, \underline{\underline{H}}-\mathrm{Ar}\right) \mathrm{ppm} ;{ }^{13} \mathrm{C} \mathrm{NMR}\left(100 \mathrm{MHz}, \mathrm{CDCl}_{3}\right) \delta 16.3$

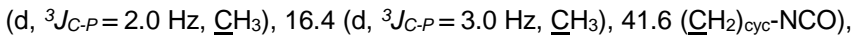
$\left.43.4\left(\underline{\mathrm{CH}}_{2}-\mathrm{Cl}\right), 45.1\left(\mathrm{CH}_{2}-\mathrm{CH}_{2}-\mathrm{Cl}\right), 48.8\left(\mathrm{CH}_{2}\right) \mathrm{cyc}^{-}-\mathrm{NCH}_{2}\right), 64.0\left(\mathrm{~d},{ }^{2} \mathrm{JC}_{\mathrm{C}-\mathrm{P}}=7.0\right.$ $\left.\mathrm{Hz}, \underline{\mathrm{C}}_{2}\right), 64.1\left(\mathrm{~d},{ }^{2} \mathrm{~J} C-\mathrm{P}=6.0 \mathrm{~Hz}, \underline{\mathrm{C}}_{2}\right), 68.5\left(\mathrm{~d},{ }^{1} \mathrm{~J} \mathrm{C}-\mathrm{P}=176.0 \mathrm{~Hz},{ }^{*} \underline{\mathrm{C}} \mathrm{H}\right)$,

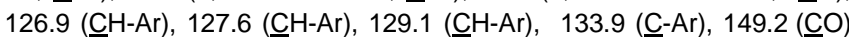
$\mathrm{ppm} ; \mathrm{MS}\left(\mathrm{ESI}^{+}\right) \mathrm{m} / \mathrm{z}=483.0[\mathrm{M}+\mathrm{Na}]^{+} ; \mathrm{HRMS}\left(\mathrm{ESI}^{+}\right): \mathrm{m} / \mathrm{z}[\mathrm{M}+\mathrm{Na}]^{+}$calcd for $\mathrm{C}_{14} \mathrm{H}_{22} \mathrm{~N}_{2} \mathrm{O}_{7} \mathrm{NaPS}_{2} \mathrm{Cl}: 483.0197$; found: 483.0192 (Diethoxyphosphoryl)(furan-2-yl) methyl 5-(2-chloroethyl)-1,2,5-
thiadiazolidine-2-carboxylate 1,1-dioxide: the product (7c, $\left.\mathrm{C}_{14} \mathrm{H}_{22} \mathrm{ClN}_{2} \mathrm{O}_{8} \mathrm{PS}\right)$ was obtained as an colorless oil $(92,42 \mathrm{mg}, 100 \%$ yield); $\mathrm{R}_{f} 0.52$ (DCM/MeOH) (9.8:0.2) ; FTIR (KBr): $1719(\mathrm{CO}), 1305$ and 1162 $\left(\mathrm{SO}_{2}\right), 1228$ and $1025(\mathrm{PO}) \mathrm{cm}^{-1}$; ${ }^{31} \mathrm{P}$ NMR $\left(161.9 \mathrm{MHz}, \mathrm{CDCl}_{3}\right) \delta 13.05$ ppm; ${ }^{1} \mathrm{H}$ NMR ( $\left.400 \mathrm{MHz}, \mathrm{CDCl}_{3}\right) \delta 1.29\left(\mathrm{q}, \mathrm{J}_{\mathrm{H}-\mathrm{H}}=7.2 \mathrm{~Hz}, 6 \mathrm{H}, 2 \mathrm{CH}_{3}\right), 3.46$ $\left(\mathrm{t}, J_{H-H}=6.8 \mathrm{~Hz}, 2 \mathrm{H},\left(\mathrm{CH}_{2}\right)_{\mathrm{cyc}}-\mathrm{NCH}_{2}\right), 3.56\left(\mathrm{t}, J_{H-H}=6.4 \mathrm{~Hz}, 2 \mathrm{H}, \underline{\mathrm{C}}_{2}-\mathrm{CH}_{2}-\right.$ $\mathrm{Cl}), 3.71\left(\mathrm{t}, \mathrm{J}-\mathrm{H}=6.4 \mathrm{~Hz},\left(\mathrm{CH}_{2}\right)_{\mathrm{cyc}}-\mathrm{NCO}\right), 3.92\left(\mathrm{t}, J_{H-H}=6.4 \mathrm{~Hz}, 2 \mathrm{H}, \mathrm{C}_{2}-\right.$ $\mathrm{Cl}), 4.18-4.25\left(\mathrm{~m}, 4 \mathrm{H}, 2 \mathrm{C}_{2}-\mathrm{CH}_{3}\right), 6.21\left(\mathrm{~d}, \mathrm{~J}_{\mathrm{H}-\mathrm{P}}=15.2 \mathrm{~Hz}, 1 \mathrm{H},{ }^{*} \mathrm{C}\right.$ H), $6.39(\mathrm{t}$, $\left.J_{H-H}=1.6 \mathrm{~Hz}, 1 \mathrm{H}, \underline{\mathrm{H}}-\mathrm{Ar}\right), 6.67\left(\mathrm{t}, J_{H-H}=2.8 \mathrm{~Hz}, 1 \mathrm{H}, \underline{\mathrm{H}}-\mathrm{Ar}\right), 7.46\left(\mathrm{t}, J_{H-H}=1.2\right.$ $\mathrm{Hz}, 1 \mathrm{H}, \underline{\mathrm{H}}-\mathrm{Ar}) \mathrm{ppm} ;{ }^{13} \mathrm{C} \mathrm{NMR}\left(100 \mathrm{MHz}, \mathrm{CDCl}_{3}\right) \delta 16.3\left(\mathrm{~d},{ }^{3} \mathrm{JC}_{\mathrm{C}} \mathrm{P}=4.0 \mathrm{~Hz}\right.$,

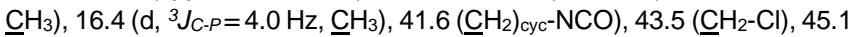
$\left.\left(\mathrm{C}_{2}-\mathrm{CH}_{2}-\mathrm{Cl}\right), 48.8\left(\mathrm{CH}_{2}\right){ }_{\mathrm{cyc}}-\mathrm{NCH}_{2}\right), 63.9\left(\mathrm{t},{ }^{2} \mathrm{~J}_{\mathrm{C}-\mathrm{P}}=7.0 \mathrm{~Hz}, 2 \underline{\mathrm{C}}_{2}\right), 65.9(\mathrm{~d}$, $\left.{ }^{1} J_{C-P}=175.0 \mathrm{~Hz},{ }^{*} \underline{\mathrm{C}} \mathrm{H}\right), 110.9(\underline{\mathrm{C}} \mathrm{H}-\mathrm{Ar}), 112.5\left(\mathrm{~d}, J_{C-P}=5.0 \mathrm{~Hz}, \underline{\mathrm{C}} \mathrm{H}-\mathrm{Ar}\right)$,

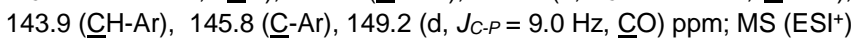
$\mathrm{m} / \mathrm{z}=467.0 \quad[\mathrm{M}+\mathrm{Na}]^{+} ;$HRMS $\left(\mathrm{ESI}^{+}\right): \mathrm{m} / \mathrm{z} \quad[\mathrm{M}+\mathrm{Na}]^{+}$calcd for $\mathrm{C}_{14} \mathrm{H}_{22} \mathrm{~N}_{2} \mathrm{O}_{8} \mathrm{NaPSCl}: 467.0419$; found: 467.0421

\subsection{Crystallographic data}

Data collection was performed, at 295(2) K, on a Bruker APEXII diffractometer, $C C D$ area detector equipped with a graphite monochromatized Mo Ka radiation $(\lambda=0.71073 \AA)$. The crystallographic data and experimental details for structural analysis are summarized in (Table S1, SI). The reported structures were solved by direct methods with $\mathrm{SIR} 2002{ }^{[31]}$ to locate all the non- $\mathrm{H}$ atoms which were refined anisotropically with SHELXL97 [32] using full matrix least squares on F2 procedure from within the WinGX ${ }^{[33]}$ suite of software used to prepare material for publication. All the $\mathrm{H}$ atoms were located in difference Fourier maps and were placed in the calculated positions and constrained to ride on their parent atoms.

\subsection{Antimicrobial activity}

Antimicrobial activity of the selected synthetic compounds $(\mathbf{2 b}, \mathbf{4 b}, \mathbf{6 b}, \mathbf{7 b}$, 1c, $3 c$ and 5c) were determined using the clinical and reference microorganisms by mean of an agar diffuse on method described by Toty et $a^{a}{ }^{[34]}$ and Demoulin et al [35] with some modifications. Briefly, the microbial cell suspensions were prepared and adjusted to an OD of 0.08 to 0.1 read at $625 \mathrm{~nm}$, referring to $10^{8}$. UFC / $\mathrm{ml}$ (CLSIS, 2007).

Each cell suspension was spread uniformly on the Mueller Hinton (MH) agar medium and incubated $20 \mathrm{~min}$ at $37^{\circ} \mathrm{C}$ allowing the formation of a homogeneous microbial mat. Following this incubation, three paper discs (Whatman No. 3) of $6 \mathrm{~mm}$ in diameter were impregnated with either $10 \mu \mathrm{l}$ of synthetic compounds $(\mathbf{2 b}, \mathbf{4 b}, \mathbf{6 b}, \mathbf{7 b}, \mathbf{1 c}, \mathbf{3 c}$ and $\mathbf{5 c})$, (test disc) and put in contact with the $\mathrm{MH}$ agar medium being previously populated with one species or another of the microorganisms. Experimental controls include acetone or antibiotic (gentamycin)/anti-fungus (amphotericin-B) impregnated discs.

The preparations were then incubated overnight at $37^{\circ} \mathrm{C}$ and each test was performed in triplicate. The absence of microbial growth results in a clear halo around the disc and the diameter of the zone of inhibition (DZI) were measured.

The solid dilution technique is used and recommended by the institute of clinical and laboratory standards (CLSI, 2007) to assess the sensitivity of micro-aerophilic bacteria to antibacterial agents. The MIC determination tests are carried out according to the standard dilution method, respectively, on semi-solid bikes of the Mueller Hinton (MHA) type for bacteria; and Saboureaud agar recommended for yeasts.

A base 2 dilution range is made, in acetone, from $50 \mu$ of our compounds to test. The range of concentrations thus published is between 1 and 0.03 $\mathrm{mg} \cdot \mathrm{mL}^{-1}$. The contents of each tube are immediately returned to a sterile Petri dish after stirring for 15 seconds. $2 \mu \mathrm{L}$ spots of an inoculum standardized to $10^{8}$ UFC. $\mathrm{mL}^{-1}$ (i.e. 2.105 UFC per spot) are deposited on the agar plates. Gentamycin / amphoterine B is used as a positive control, acetone is used as a negative control. The dishes are incubated for $24 \mathrm{~h}$ at $37^{\circ} \mathrm{C}$. The MIC of the synthetic molecules is defined from the first dish in the range devoid of bacterial growth. The test is carried out in three successive technical repetitions.

\section{Acknowledgements}

We extend our gratitude to General Directorate for Scientific Research and Technological Development (DG-RSDT), Algerian Ministry of Scientific Research and also PRFU Project (2021; ref (B00L01UN230120200006). We would like to thank CEISAM laboratory of Nantes University and the AMaCC platform staff for their help in the identification of our products.

Keywords: Cyclosulfamide $\bullet$ Phosphonate $\cdot$ Chlorosulfonyl isocyanate $\cdot$ Antibacterial activity $\cdot \mathrm{XR}$ study

[1] A. J. Zych, S. Q. Lam, D. M. Jenkins, R. J. Herr, P. C. Ting, J. F. Lee, R. Kuang, H. Wu, D. W. Kim, R. G. Aslanian, S. Wainhaus, T. A. Black, A. Cacciapuoti, P. M. McNicholas, Y. Xu, S. S. Walker, Bioorg. Med. Chem. Lett. 2012, 22, 4896-4899.

[2] B. Özgeriş, S. Göksu, L. P. Köse, İ. Gülçin, R. E. Salmas, S. Durdagi, F. Tümer, C. T. Supuran, Bioorg. Med. Chem. 2016, 24, 2318-2329.

[3] B. Ozgeris, Y. Akbaba, O. Ozdemir, H. Türkez, S. Göksu, Arch. Med. Res. 2017, 48, 513-519.

[4] J. D. Park, D. H. Kim, S. J. Kim, J. R. Woo, S. E. Ryu, J. Med. Chem 2002, 45, 5295-5302.

[5] J. Y. Winum, A. Scozzafava, J. L. Montero, C.T. Supuran, Med. Res. Rev. 2006, 26, 767-792.

[6] J. Y. Winum, A. Scozzafava, J. L. Montero, C.T. Supuran, Expert Opin. Ther. Pat. 2006, 16, 27-47. 
[7] A. Ax, W. Schaal, L. Vrang, B. Samuelsson, A. Hallberg, A. Karlen, Bioorg. Med. Chem. 2005, 13, 755-764.

[8] J. J. Jun, X. Q. Xie, ChemistrySelect. 2021, 6, 430-469.

[9] G. Ahlsén, J. Hultén, C. F. Shuman, A. Poliakov, M. T. Lindgren, M. Alterman, B. Samuelsson, A. Hallberg, U. H. Danielson, Antivir. Chem. Chemother. 2002, 13, 27-37.

[10] J. J. Jun, D. Duscharla, R. Ummanni, P. R. Hanson, S. V. Malhotra, ACS. Med. Chem. Lett. 2021, 12, 202-210.

[11] S. J. Kim, M-H. Jung, K. H. Yoo, J-H. Cho, C-H. Oh, Bioorg. Med. Chem. Lett. 2008, 18, 5815-5818.

[12] A. Spaltenstein, M. R. Almond, W. J. Bock, D. G. Cleary, E. S. Furfine, R. J. Hazen, W. M. Kazmierski, F. G. Salituro, R. D. Tung, L. L. Wright, Bioorg. Med. Chem. Lett. 2000, 10, 1159-1162.

[13] T. Sparey, D. Beher, J. Best, M. Biba, J. L. Castro, E. Clarke, J. Hannam, T. Harrison, H. Lewis, A. Madin, M. Shearman, B. Sohal, N. Tsou, C. Welchd, J. Wrigley, Bioorg. Med. Chem. Lett. 2005, 15, 4212-4216.

[14] D. Dou, S. R. Mandadapu, K. R. Alliston, Y. Kim, K-O. Chang, W. C. Groutas, Eur. J. Med. Chem. 2012, 47, 59-64.

[15] J. Joossens, O. Mouhamed-Ali, I. El-Sayed, G. Surpateanu, P. Van der Veken, A.-M. Lambeir, B. Setyono-Han, J. A. Foekens, A. Schneider, W Schmalix, A. Haemers, K. Augustyns, J. Med. Chem. 2007, 50, 6638 6646.

[16] Z. Chen, W. Xu, K. Liu, S. Yang, H. Fan, P. S. Bhadury, D-Y. Huang, Y. Zhang, Molecules. 2010, 15, 9046-9056.

[17] N. Alimoradi, M. R. Ashrafi-Kooshk, M. Shahlaei, S. Maghsoudi, H. Adibi, R. P. McGeary, R. Khodarahmi, J. Enzyme Inhib. Med. Chem. 2017, 32 20-28.

[18] R. Karpowicz, J. Lewkowski, M. Stasiak, A. Czopor, P. Tokarz, A. Król, D. Rogacz, P. Rychter, Phosphorus, Sulfur Relat. Elem. 2018, 193, 423436.

[19] S. Bhagat, M. Supriya, S. Pathak, D. Sriram, A. K. Chakraborti, Bioorg. Chem. 2019, 82, 246-252.

[20] K. Bechlem, M. Aissaoui, B. Belhani, K. Otmane Rachedia, S. Bouacida, R. Bahadi, S-E., Djouad, R. Ben Mansour, M. Bouaziz, F. Almalki, T. Ben Hadda, M. Berredjem, J. Mol. Struct. 2020, 1210, 127990-128016.
[21] J. Y. Winum, J. L. Bouissière, I. Passagne, A. Evrard, V. Montero, P. Cuq J. L. Montero, Eur. J. Med. Chem. 2003, 38, 319-324.

[22] H. Timmler, R. Wegler, G. Unterstenhafer, Procédé de Fabrication de Dérivés Phosphorylés de Sulfonyle Urethanes, FR1, 403, 523A, 18 June 1965.

[23] A. Bouzina, K. Bechlem, H. Berredjem, B. Belhani, I. Becheker, J. Lebreton, M. Le Borgne, Z. Bouaziz, C. Marminon, M. Berredjem, Molecules. 2018, 23, 1682-169.

[24] B. Belhani, M. Berredjem, M. Le Borgne, Z. Bouaziz, J. Lebreton, N. E. Aouf, RSC. Adv. 2015, 5, 39324-39329.

[25] A. Bouzina, B. Belhani, N-E. Aouf, M. Berredjem, RSC. Adv. 2015, 5, $46272-46275$.

[26] S. Hessainia, M. Berredjem, S. Ouarna, Z. Cheraiet, N-E. Aouf, Phosphorus, Sulfur Silicon Relat. Elem. 2013, 188, 719-725.

[27] H. K'tir, Z. Aouf, T. O. Souk, R. Zerrouki, M. Berredjem, N-E. Aouf, Phosphorus, Sulfur Silicon Relat. Elem. 2017, 192, 555-559.

[28] a) S. Boughaba, Z. Aouf, O. Bechiri, M. Mathe-Allainmat, J. Lebreton, N E. Aouf, Phosphorus, Sulfur, Silicon Relat. Elem. 2021,196, 28-35. b) S Boughaba, S. Bouacida, Z. Aouf, O. Bechiri, N-E.Aouf, Curr. Org. Chem. 2018, 22, 1335-1341. c) A. Amira, Z. Aouf, H. K'tir, Y. Chemam, R Ghodbane, R. Zerrouki, N - E. Aouf, ChemistrySelect. 2021, 6, 6137 6149

[29] V. Abramov, Dokl. Akad. Nauk SSSR. 1950, 73, 487-489.

[30] Z. Aouf, S. Boughaba, S. Lakrout, O. Bechiri, N-E. Aouf, Chem. Chem. Technol. 2020, 14,154-160.

[31] M.C. Burla, R. Caliandro, M. Camalli, B. Carrozzini, G.L.Cascarano, L. De Caro, C. Giacovazzo, G. Polidori, R. Spagna, J. Appl. Crystallogr. 2005, 38, 381-388.

[32] G. M. Sheldrick, J. Acta Cryst. 2008, 64, 112-122.

[33] L. J. Farrugia, J. Appl. Cryst. 2012, 45, 849-854.

[34] A. A. Toty, N. Guessennd, C. Bahi, A. M. Kra, D. A. Otokore, M. Dosso, Bull. Soc. r. sci. Liège. 2013, 82, 12-21.

[35] L. Demoulin, M. Medard, J. Kellens, J. Kellens, Pathol. Biol. 1982, 30 201-205. 


\section{Entry for the Table of Contents}

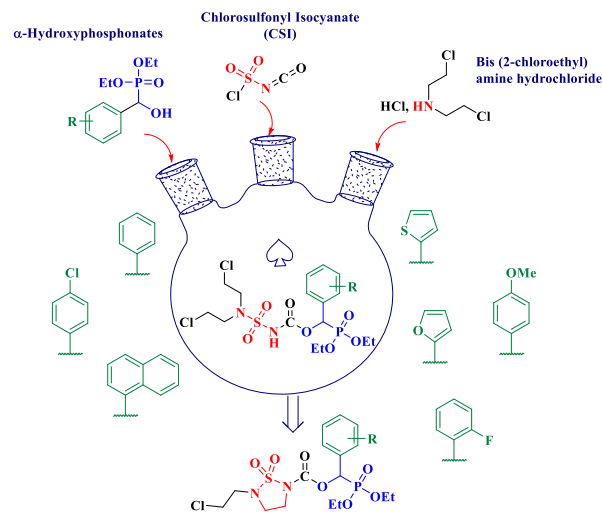

Seven $N, N$-bis (2-chloroethyl) sulfamidocarbonyloxymethylphosphonate derivatives and their cyclic compounds were synthesized in three steps (Abramov reaction, carbamoylation-sulfamoylation with CSI reagent and intramolecular cyclisation). A single crystal of compound $3 c$ was designated for single crystal X-ray diffraction analysis. Some compounds were selected to examine the antimicrobial activity against clinical and reference microorganisms. 